\title{
Resolving the mid-infrared cores of local Seyferts ${ }^{\star}$
}

\author{
P. Gandhi ${ }^{1}$, H. Horst ${ }^{2,3,4}$, A. Smette ${ }^{5}$, S. Hönig ${ }^{4}$, A. Comastri ${ }^{6}$, R. Gilli ${ }^{6}$, C. Vignali ${ }^{7}$, and W. Duschl ${ }^{2}$ \\ 1 RIKEN Cosmic Radiation Lab, 2-1 Hirosawa, Wakoshi, Saitama 351-0198, Japan \\ e-mail: pg@crab.riken.jp \\ 2 Institut für Theoretische Physik und Astrophysik, Christian-Albrechts-Universität zu Kiel, Leibnizstr. 15, 24098 Kiel, Germany \\ 3 Zentrum für Astronomie, ITA, Universität Heidelberg, Albert-Ueberle-Str. 2, 69120 Heidelberg, Germany \\ 4 Max-Planck-Institut für Radioastronomie, Auf dem Hügel 69, 53121 Bonn, Germany \\ European Southern Observatory, Alonso de Cordova 3107, Casilla 19001, Santiago, Chile \\ 6 Istituto Nazionale di Astrofisica (INAF) - Osservatorio Astronomico di Bologna, via Ranzani 1, 40127 Bologna, Italy \\ 7 Dipartimento di Astronomia, Università degli Studi di Bologna, via Ranzani 1, 40127 Bologna, Italy
}

Received 17 November 2008 / Accepted 6 February 2009

\section{ABSTRACT}

\begin{abstract}
We present new photometry of 16 local Seyferts including 6 Compton-thick sources in $N$-band filters around $12-\mu$ m, obtained with the VISIR instrument on the 8-m Very Large Telescope. The near-diffraction-limited imaging provides the least-contaminated core fluxes for these sources to date. Augmenting these with our previous observations and with published intrinsic X-ray fluxes, we form a total sample of 42 sources for which we find a strong mid-infrared:X-ray $(12.3 \mu \mathrm{m}: 2-10 \mathrm{keV})$ luminosity correlation. Performing a physically-motivated subselection of sources in which the Seyfert torus is likely to be best-resolved results in the correlation $L_{\mathrm{MIR}} \propto$ $L_{\mathrm{X}}{ }^{1.11 \pm 0.07}$, with a reduction of the scatter in luminosities as compared to the full sample. Consideration of systematics suggests a range of 1.02-1.21 for the correlation slope. The mean $2-\mathrm{keV}: 12.3-\mu \mathrm{m}$ spectral index $\left(\alpha_{\mathrm{IX}}\right)$ is found to be $-1.10 \pm 0.01$, largely independent of luminosity. Indirectly-computed $12-\mu \mathrm{m}$ bolometric corrections range over $\approx 10-30$ if a known luminosity trend of X-ray bolometric corrections is assumed. Comparison with ISO data spanning a wider luminosity range suggests that our correlation can be extended into the quasar regime. That unobscured, obscured, and Compton-thick sources all closely follow the same luminosity correlation has important implications for the structures of Seyfert cores. The typical resolution-limit of our imaging corresponds to $\sim 70 \mathrm{pc}$ at a median $z=0.01$, and we use the tightness of the correlation to place constraints on the dominance of any residual emission sources within these physical scales. An upper-limit for any contaminating star formation of $\approx 40 \%$ of the unresolved flux is inferred, on average. We suggest that uncontaminated mid-IR continuum imaging of AGN is an accurate proxy for their intrinsic power.
\end{abstract}

Key words. galaxies: active - infrared: galaxies - X-rays: galaxies - galaxies: Seyfert - accretion, accretion disks

\section{Introduction}

Typical broad-band spectra of active galactic nuclei (AGN) are known to show a significant bump in the infrared regime (e.g. Barvainis 1987; Sanders et al. 1989; Elvis et al. 1994, and references therein). This is thought to originate in the absorption of intrinsic AGN ultraviolet, X-ray, and optical radiation by dusty clouds in the AGN "torus" on $\sim$ pc-scales, which subsequently radiate this energy at infrared (IR) frequencies. But isolating the AGN emission from other ubiquitous contaminating sources, such as bright starbursts within the inner few kpc of AGN host galaxies (e.g. Rodriguez Espinosa et al. 1987; Norman \& Scoville 1988; González Delgado et al. 2001), is difficult because of the finite resolving powers of IR observatories. Telescopes with improving sensitivity, wavelength coverage, and resolving power have changed the situation steadily over the past two decades (e.g., Sanders et al. 1989; Mulchaey et al. 1994; Spinoglio et al. 1995; Krabbe et al. 2001; Lutz et al. 2004; Buchanan et al. 2006; Ramos Almeida et al. 2007; Mushotzky et al. 2008; Meléndez et al. 2008; McKernan et al. 2009). These works find increasingly stronger correlations between the measured IR powers with the intrinsic (X-ray, bolometric, or various forbidden emission line) AGN luminosities, but still with significant scatter. How much of this scatter is inherent to the physical

^ Based on observations carried out in ESO programme 080.B-0860. conditions of the AGN and torus clouds, as compared to observational selection effects, remains an important unresolved issue.

In two papers (Horst et al. 2006, 2008, hereafter Papers I and II), some of us presented high-resolution imaging of nearby AGN with the VLT/VISIR mid-infrared camera (Lagage et al. 2004) in filters covering the $8-13 \mu \mathrm{m}$ atmospheric transmission window. The diffraction limit of the $8.2 \mathrm{~m}$-diameter mirrors of the VLT over this $N$-band window is $\approx 00^{\prime} 3-0.0^{\prime} 4$, which is matched to the mid-IR seeing at the VLT site under reasonable optical seeing conditions; thus, VISIR $N$-band imaging is usually diffraction-limited. A sample of about 30 local AGN were imaged in these two papers. Because of the unprecedented angular resolution, the unresolved core fluxes are the best ones (i.e. least biased by extra-nuclear emission) available for these objects. In a follow-up work, Horst et al. (2009, hereafter Paper III) compared VISIR multi-filter photometric spectral energy distributions (SEDs) with sensitive Spitzer IRS spectra of the same sources and quantified the level of contamination by extended emission, finding non-negligible contamination in Spitzer observations in about $40 \%$ of the cases.

By extracting intrinsic X-ray luminosities $\left(L_{2-10 \mathrm{keV}}\right.$, hereafter $L_{\mathrm{X}}$ ) from the recent literature, we were able to compare these with the IR powers measured to find a good correlation between these quantities (see, e.g., Fig. 1 in Paper II). The main results of our works are that $i$ ) the dispersion in the mid-IR:X-ray 
correlation is less than found in previous works and ii) both Seyfert-1s and Seyfert-2s closely follow the same correlation. This second result was not predicted by radiative transfer models in which intrinsic AGN emission is re-processed by obscuring tori with smoothly-distributed dust. This occurs because an optically-thick line-of-sight through the torus will primarily show cooler dust and a lower mid-infrared luminosity for the same $L_{X}$ than does an optically-thin one (see, e.g., Fig. 1 of Pier \& Krolik 1993). Result ii) above was also found in previous studies with lower-resolution ground and space observatories (e.g., Krabbe et al. 2001; Lutz et al. 2004), but was ascribed to the expected differences being mitigated by unresolved contamination and other selection biases. On the other hand, we found a similar result in our high-resolution VLT imaging, even though contamination is much less severe. This suggested, instead, that dust clouds have significant clumpiness in the AGN tori (e.g., Dullemond \& van Bemmel 2005; Hönig et al. 2006; Nenkova et al. 2008a,b; Schartmann et al. 2008), which leads to the observed mid-IR emission from hot illuminated cloud faces in the inner torus regions being largely isotropic. The extent to which the mid-IR emission is isotropic can be determined by quantifying the scatter and offsets of obscured and unobscured AGN at any given $L_{X}$ in the mid-IR:X-ray correlation. Our study has so far been restricted to sources with low obscuring column densities of cold gas. But the differences between the expectations of clumpy and smooth torus distributions increase with line-ofsight optical depth, so it is important to obtain high-resolution mid-IR imaging of highly obscured sources.

Even irrespective of the question of torus physical structure, an accurate census of local Seyferts in the mid-IR is important for determining their bolometric accretion energetics and disentangling AGN from starburst emission. If, after removing all selection biases, the scatter in the correlation between IR and intrinsic AGN power can be reduced, then the hope is that mid-IR observation may be an independent isotropic indicator of AGN energetics, similar to forbidden [OIII] $\lambda 5007 \AA$ emission-line, far-infrared, hard X-ray, or low-frequency radio observations (cf. Mulchaey et al. 1994).

In this paper, we present new high-resolution mid-IR fluxes around $12-\mu \mathrm{m}$ of 16 local Seyferts, including 7 sources obscured by high Compton-thin absorption (with $N_{\mathrm{H}}>10^{23} \mathrm{~cm}^{-2}$ ) and 6 Compton-thick ones $\left(N_{\mathrm{H}}>1.5 \times 10^{24} \mathrm{~cm}^{-2}\right)$. Adding literature observations of another well-studied Compton-thick source NGC 1068, as well as our previous sample of 25 detections from Paper II, and comparing these unbiased core mid-IR measurements with the best $L_{\mathrm{X}}$ values from the literature, we find the luminosity relationship to be very tight, especially for the best resolved targets. Various implications for the core structures of nearby AGN are discussed and useful broad-band (mid-IR to X-ray) spectral relations are derived. A flat Universe with a Hubble constant $H_{0}=71 \mathrm{~km} \mathrm{~s}^{-1} \mathrm{Mpc}^{-1}, \Omega_{\Lambda}=0.73$ and $\Omega_{\mathrm{M}}=0.27$ is assumed. All literature measurements have been converted to this cosmology.

\section{Observations}

A sample of 16 targets was observed with VISIR during service nights spanning the semester from 2007 October to 2008 March. These were mainly selected from sources that had been observed (or had planned forthcoming observations) with hard X-ray satellites including Suzaku, INTEGRAL and Swift, so that their broad-band X-ray spectra could be modeled and $2-10 \mathrm{keV}$ AGN luminosities accurately determined; i.e. the sample is not complete. The sky conditions were required to be clear with 0.'8 optical seeing or less, during ESO period 80. Observational and data reduction procedures are very similar to those described in Paper II, so only minimal details are stated here. A standard parallel chopping and nodding technique with a chop throw of $8^{\prime \prime}$ was used. Observations were carried out with the small field camera $\left(0{ }^{\prime}(075 /\right.$ pixel $)$ in one or more narrow and intermediate-band filters covering the $N$-band, for each source. As we are interested in determining source continuum luminosities, filters were selected to avoid emission lines and to include continuum regions free of the silicate absorption trough, depending on source redshift.

The science and standard photometric star calibration frames were reduced with a dedicated pipeline (Pantin et al. 2005), including removal of detector features and background subtraction. The count rate for one full exposure was calculated as the mean of the 3 beams from all nodding cycles of this exposure. To minimize the effect of residual sky background, we chose relatively-small apertures $(\approx 10$ pixels $=0.75)$ for the photometry of most sources and corrected the count rates using the radial profiles of standard stars. For a handful of faint targets, profile-fitting (based on the profiles of bright standard stars) on binned images was carried out instead. As an initial error estimate, the standard deviation of the fluxes amongst the three nodding beams was used, except for a few cases where the statistical error on source counts turned out to be greater. The absolute flux uncertainty is dependent on the counts-to-flux "conversion" factors determined from standard star observations and the known accuracy of stellar templates. For the PAH2 filter, the conversion factor has been found to be constant to within $\approx 5 \%$ over a whole observing period, and this minimum uncertainty is added in quadrature to the initial error estimate for all sources.

The X-ray data used in this paper were carefully selected (or computed) from recent published studies including spectral modeling over broad energy ranges where available. The $L_{X}$ values were corrected for obscuration, complex spectral components, and redshift effects, so should be a measure of the true, intrinsic 2-10 keV AGN power responsible for heating torus clouds. Of course, in cases of extreme obscuration or complexity, these corrections can have large uncertainties - see Sect. 6. In this respect, it will be useful to compare against hard X-ray luminosities measured above $10 \mathrm{keV}$ (cf. Mushotzky et al. 2008) in future work, as these should be less susceptible to such complexities. Since data from a variety of published sources have been compiled for each source, the error assignment on $L_{X}$ is necessarily somewhat loose. As in Papers I and II, the uncertainty is based on the range of peak-to-peak published values, which should include source variability and other biases. Each source is detailed in the Appendix.

\section{Results}

All 16 sources were detected in filters around $12-\mu \mathrm{m}$, and the images were dominated in each case by a single IR AGN core. The presentation of images and discussion of full multi-filter $\mathrm{N}$-band properties is not the purpose of the present work. We refer any reader interested in the general high spatial-resolution Seyfert morphologies to our Paper III, which includes our entire previous sample. For the purpose of the IR:X-ray luminosity correlation (cf. Papers I and II), the $12.3-\mu \mathrm{m}$ source flux is the important quantity. We therefore concentrate mainly on the filter most appropriate for probing the continuum closest to $12.3 \mu \mathrm{m}$. Table 1 lists all the observational parameters of our sample, as follows: Col. (1) source name, tabulated in order of increasing right ascension except for the extra target NGC 1068 
Table 1. Observed properties of the sample.

\begin{tabular}{|c|c|c|c|c|c|c|c|c|c|c|c|c|}
\hline \multirow{2}{*}{$\begin{array}{l}\text { Target } \\
\text { (1) }\end{array}$} & \multirow{2}{*}{$\begin{array}{l}\text { Sy } \\
\text { (2) }\end{array}$} & \multirow{2}{*}{$\begin{array}{l}z \\
(3)\end{array}$} & \multirow{2}{*}{$\begin{array}{c}\log N_{\mathrm{H}} \\
\mathrm{cm}^{-2} \\
(4)\end{array}$} & \multirow{2}{*}{$\begin{array}{l}r_{0} \\
\mathrm{pc} \\
(5)\end{array}$} & \multicolumn{3}{|c|}{ Secondary filter flux } & \multicolumn{3}{|c|}{ Primary filter flux } & \multirow{2}{*}{$\begin{array}{c}\log \lambda L_{\lambda} \\
\mathrm{erg} \mathrm{s}^{-1} \\
(10)\end{array}$} & \multirow{2}{*}{$\begin{array}{r}\log L_{X} \\
\mathrm{erg} \mathrm{s}^{-1} \\
(11)\end{array}$} \\
\hline & & & & & Name & $\lambda_{\mathrm{C}}$ & $\begin{array}{c}\text { mJy } \\
(7)\end{array}$ & $\begin{array}{r}\text { Name } \\
(8\end{array}$ & $\lambda_{\mathrm{C}}$ & $\begin{array}{c}\mathrm{mJy} \\
(9)\end{array}$ & & \\
\hline NGC 1068 & lh & $14.4^{d}$ & $>25$ & 25 & & - & & $\approx 12$ & $i m^{*}$ & $\sim 9500$ & $43.80 \pm 0.02$ & $43.4 \pm 0.3$ \\
\hline Swift J0138.6-4001 & 2 & 0.0252 & 23.7 & 175 & SIV & 10.49 & $20 \pm 3$ & PAH2 & 11.25 & $34 \pm 3$ & $43.10 \pm 0.04$ & $43.6 \pm 0.3$ \\
\hline Swift J0601.9-8636 & 2 & 0.0062 & 24.0 & 45 & $\mathrm{PAH} 2$ & 11.25 & $13 \pm 4$ & NEII_2 & 13.04 & $72 \pm 15$ & $42.11 \pm 0.08$ & $41.9 \pm 0.3$ \\
\hline ESO 209-G012 & 1.5 & 0.0405 & $<22$ & 280 & PAH2 & 11.25 & $162 \pm 10$ & NEII & 12.81 & $188 \pm 26$ & $44.22 \pm 0.06$ & $43.7 \pm 0.2$ \\
\hline NGC 3081 & $1 \mathrm{~h}$ & 0.007 & 23.8 & 55 & $\mathrm{PAH} 2$ & 11.25 & $161 \pm 11$ & NEII_2 & 13.04 & $138 \pm 11$ & $42.64 \pm 0.03$ & $42.6 \pm 0.3$ \\
\hline ESO 26 & 2 & 0.03 & 23.6 & 225 & SIC & 11. & $45 \pm 4$ & NEII & 12.81 & $46 \pm 3$ & $3.42 \pm 0.02$ & $3.3 \pm 0.4$ \\
\hline NG & 2 & & 24.3 & 75 & PAH2 & 11 & $481 \pm 2$ & NEII_2 & 13. & $1016=$ & .02 & \pm 0.2 \\
\hline NGC & 2 & 0.01 & 24.2 & 90 & SIV & 10.49 & $36 \pm 2$ & NEII_2 & 13.04 & $94 \pm 5$ & $42.90 \pm 0.02$ & $43.0 \pm 0.3$ \\
\hline NGC & $1 \mathrm{~h}$ & & 23.4 & 30 & $\mathrm{PAH} 2$ & 11.25 & $7 \pm$ & NEII_2 & & $375 \pm 28$ & .03 & $42.2 \pm 0.3$ \\
\hline IC 3 & $1 \mathrm{~h}$ & 0 . & $>25$ & 75 & PAH2 & 11.25 & $326 \pm 17$ & NEII_2 & & $542 \pm 29$ & .02 & $43.6 \pm 0.5$ \\
\hline ESO & 1.9 & & - & 110 & & - & & PAH2 & 11.25 & $61 \pm 6$ & 42.96 & $42.4 \pm 0.4$ \\
\hline NGC 4 & 2 & 0.025 & 23.7 & 175 & PAH1 & 8.59 & $61 \pm 6$ & NEII & 12.81 & $71 \pm 4$ & $43.36 \pm 0.02$ & $43.2 \pm 0.3$ \\
\hline NGC 5 & 1.9 & 009 & 24.3 & 65 & & - & & NEII_2 & 13.04 & $123 \pm 10$ & $42.60 \pm 0.03$ & $42.8 \pm 0.3$ \\
\hline ESO 1 & 2 & 0.0091 & $>24.3$ & 65 & PAH2 & 11.25 & $776 \pm 40$ & NEII_2 & 13.04 & $862 \pm 45$ & $43.54 \pm 0.02$ & $43.0 \pm 0.3$ \\
\hline NGC 6 & 2 & 003 & 23.3 & 25 & PAH2 & 11.25 & $278 \pm 14$ & NEII_2 & 13.04 & $727 \pm 38$ & $42.69 \pm 0.02$ & $42.3 \pm 0.3$ \\
\hline ESO & 2 & 013 & 23.3 & 95 & SIV & 10.49 & $243 \pm 15$ & NEII_2 & 13.04 & $622 \pm 49$ & $43.74 \pm 0.03$ & $43.4 \pm 0.2$ \\
\hline NGC 6814 & 1.5 & 0.0052 & $<20.5$ & 35 & PAH2 & 11.25 & $99 \pm 6$ & NEII_2 & 13.04 & $96 \pm 6$ & $42.07 \pm 0.02$ & $42.1 \pm 0.4$ \\
\hline
\end{tabular}

${ }^{d}$ Distances in Mpc. * For NGC 1068, the fluxes are from high angular resolution (0!'2-0!'4) Keck and Subaru studies (Mason et al. 2006; Tomono et al. 2001) at slightly-differing wavelengths. All values for this source are written in italic font simply to draw attention to the fact that the observations are entirely drawn from other publications. See Appendix.
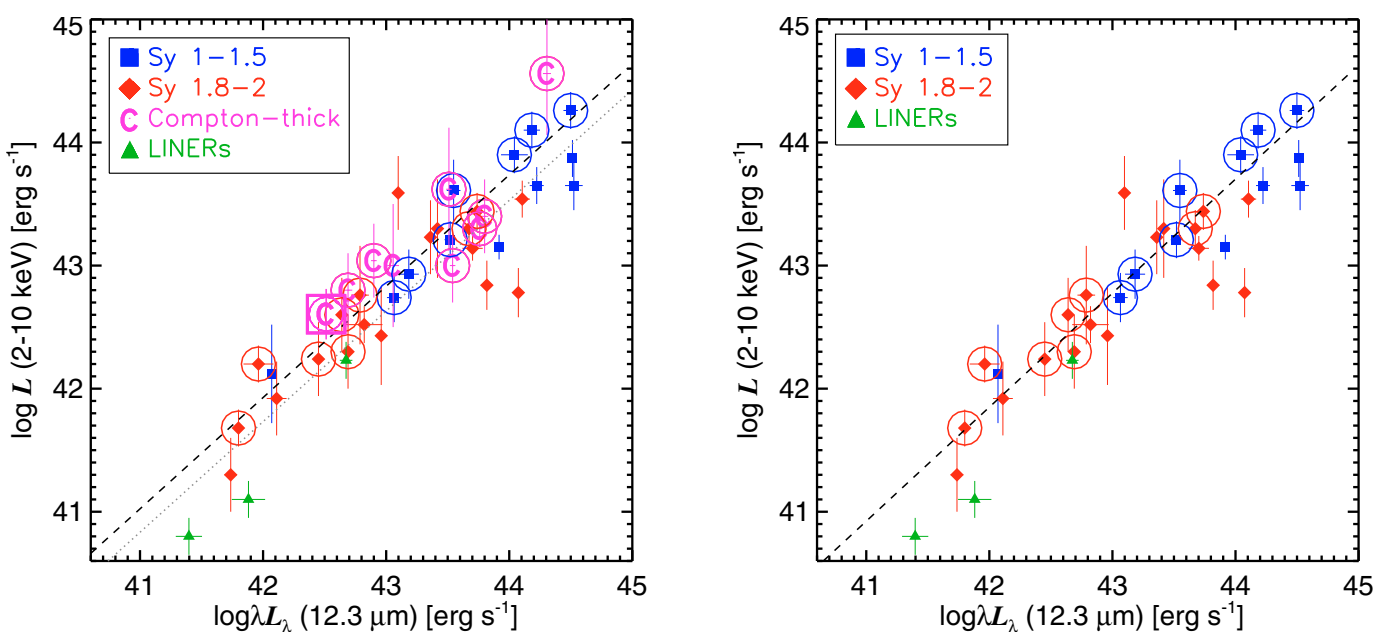

Fig. 1. Left: mid-infrared:X-ray luminosity correlation for the sample of 42 VISIR detections from Table 1 and Paper II. One additional object plotted (to make 43 in all) is ESO 428-G014 (the "C" with both a box and a circle around it), which is not part of our fit sample, but used to validate the correlation in the Compton-thick regime (see Sect. 6). The sources classified as being "well-resolved" are circled, and the diagonal dashed line is the fitted correlation for these (Eq. (2) and Table 2). The dotted grey line is the correlation fit to all 42 sources. Right: $L_{\mathrm{MIR}}$-vs.- $L_{\mathrm{X}}$ for the 34 Compton-thin sources only, with the correlation fitted to 15 well-resolved sources (Table 2).

(see below); Col. (2) Seyfert type, mostly according to VéronCetty \& Véron (2006), except for the two Swift targets, ESO 323-G032 and NGC 4992; see Appendix; Col. (3) redshift $z$; Col. (4) obscuring gas column density in units of log $\left[\mathrm{cm}^{-2}\right]$ taken from literature X-ray spectral fits; Col. (5) approximate resolved scale limit in pc, for an angular resolution of 0.35 ; Col. (6) name and central wavelength $\left(\lambda_{\mathrm{C}}\right)$ in $\mu \mathrm{m}$ of a secondary filter chosen to test systematic variations in the $L_{\mathrm{MIR}}-L_{\mathrm{X}}$ correlation (details in Sect. 5.3); Col. (7) secondary filter flux; Col. (8) chosen primary VISIR filter name and central wavelength in $\mu \mathrm{m}$; Col. (9) observed source core flux in primary filter; Col. (10) monochromatic luminosities at the central filter wavelength, in units of $\lambda L_{\lambda}$ (hereafter $L_{\mathrm{MIR}}$ ) expressed in erg s${ }^{-1}$. We assume a flat mid-IR SED in these units, i.e. no $k$-correction to $12.3 \mu \mathrm{m}$; instead, $\lambda L_{\lambda}^{12.3}$ of a source that has an SED flat in $L_{\lambda}$ units, say, will be underestimated by only 12 per cent $(0.05 \mathrm{dex})$ in the worst case; Col. (11) absorption-corrected 2-10 keV $\mathrm{X}$-ray luminosity, compiled from the literature. In addition to our 16 sources, we included literature fluxes of NGC 1068, as it is one of the most thoroughly studied nearby AGN. The full data set from Papers I, II and herein is collected in Table B.1 in the Appendix.

Figure 1 shows the correlation between $L_{\mathrm{X}}$ and $L_{\mathrm{MIR}}$. All 25 VISIR detections from Paper II and the 17 measurements from Table 1 are plotted and split into Seyfert 1-1.5 (12 objects, hereafter "Sy 1"), Seyfert 1.8-2 or 1 h (19 objects, hereafter "Sy 2"), LINERs (3 objects), and Comptonthick (8 objects) AGN. The "1 h" subclass of Sy 2 s refers to sources with broad optical emission detected in polarized light (Véron-Cetty \& Véron 2006). Since Compton-thick sources (with $N_{\mathrm{H}} \geq 1.5 \times 10^{24} \mathrm{~cm}^{-2}$ ) are of special interest, we chose to plot them with a separate symbol, though all of them fall into the 
Table 2. Correlation properties between $\log L_{\mathrm{X}}$ and $\log L_{\mathrm{MIR}}$ to various subsample populations.

\begin{tabular}{lcccccr}
\hline \hline Subsample & $N$ & $\rho_{\cdot}$ & $a$ & $b$ & $\bar{r}$ & $\sigma_{r}$ \\
$(1)$ & $(2)$ & $(3)$ & $(4)$ & $(5)$ & $(6)$ & $(7)$ \\
\hline All & $\mathbf{4 2}$ & $\mathbf{0 . 8 1}$ & $\mathbf{0 . 4 1} \pm \mathbf{0 . 0 3}$ & $\mathbf{1 . 1 1} \pm \mathbf{0 . 0 4}$ & $\mathbf{0 . 3 0}$ & $\mathbf{0 . 3 6}$ \\
All, excluding CT & 34 & 0.79 & $0.43 \pm 0.04$ & $1.11 \pm 0.04$ & 0.35 & 0.36 \\
All Sy 1 & 12 & 0.71 & $0.34 \pm 0.09$ & $1.18 \pm 0.14$ & 0.34 & 0.31 \\
All Sy 2 + CT & 27 & 0.77 & $0.40 \pm 0.06$ & $1.40 \pm 0.11$ & 0.25 & 0.38 \\
& & & & & & \\
Well-resolved & $\mathbf{2 2}$ & $\mathbf{0 . 8 9}$ & $\mathbf{0 . 1 9} \pm \mathbf{0 . 0 5}$ & $\mathbf{1 . 1 1} \pm \mathbf{0 . 0 7}$ & $\mathbf{0 . 1 5}$ & $\mathbf{0 . 2 3}$ \\
Well-resolved, excluding CT & 15 & 0.93 & $0.18 \pm 0.05$ & $1.08 \pm 0.07$ & 0.17 & 0.18 \\
Less-resolved & 20 & 0.76 & $0.62 \pm 0.05$ & $1.11 \pm 0.06$ & 0.47 & 0.40 \\
& \multicolumn{7}{c}{ Systematic variations } & & & \\
Well-resolved (Systematic 1 $\uparrow)$ & 25 & 0.82 & $0.25 \pm 0.05$ & $1.21 \pm 0.08$ & 0.17 & 0.30 \\
Well-resolved (Systematic 1 $\downarrow)$ & 16 & 0.90 & $0.26 \pm 0.05$ & $1.07 \pm 0.08$ & 0.21 & 0.23 \\
Well-resolved (Systematic 2) & 22 & 0.83 & $0.22 \pm 0.05$ & $1.09 \pm 0.07$ & 0.16 & 0.27 \\
Well-resolved (Systematic 3) & $*$ & $*$ & $1.87 \pm 0.31$ & $1.11 \pm 0.10$ & $*$ & $*$ \\
Well-resolved (Systematic 4) & $*$ & $*$ & $0.15 \pm 0.05$ & $1.02 \pm 0.07$ & $*$ & $*$ \\
All (Systematic 5) & 42 & 0.74 & $0.42 \pm 0.03$ & $1.11 \pm 0.04$ & 0.30 & 0.44 \\
Well-resolved (Systematic 5) & 22 & 0.78 & $0.21 \pm 0.05$ & $1.09 \pm 0.07$ & 0.13 & 0.31 \\
\hline
\end{tabular}

"CT" refers to Compton-thick sources. The two solutions likely to be of most interest (see Sect. 7) are written in bold. The final seven rows refer to tests for systematic variations in the correlation; see Sects. 5.1-5.3. ${ }^{\dagger}$ This test (Systematic 3) is carried out in linear (not log) luminosity space (see Sect. 5.2). * For Systematics 3 and 4, the subsamples are identical to the main well-resolved sample of 22 sources, and so the parameters marked with a star are identical as well.

optical "Sy 2" category. In addition to these 42 sources, one additional Compton-thick object (ESO 428-G014) is plotted with a box around it. This is not part of the correlation fit, but is used as an aposteriori check and is discussed in Sect. 6.

As discussed in Paper II, and shown in Table 1, the sample covers a wide range (about a factor 40) of spatial scales at the mid-IR angular resolution limit $\left(\theta_{0}\right)$ of the VLT. To make a physically-meaningful selection, Horst et al. (2008) chose only those sources in which the resolved scale $\left(r_{0}\right)$, in units of the size of the dust sublimation radius $\left(r_{\text {sub }}\right)$, lie below a certain threshold. By comparing $L_{\mathrm{MIR}}$ to $L_{\mathrm{X}}$ over the range of resolved scales, they identified a value $r_{0} / r_{\text {sub }}=560$ beyond which the ratio $L_{\mathrm{MIR}} / L_{\mathrm{X}}$ exhibited a sharp increase in its mean value and dispersion. A constant $\theta_{0}=0.35$ was assumed, and the relation

$r_{\mathrm{sub}}=0.5 \sqrt{L_{\mathrm{Bol}}^{45}} \mathrm{pc}$

was used, where $L_{\mathrm{Bol}}^{45}$ is the source bolometric luminosity in units of $10^{45} \mathrm{erg} \mathrm{s}^{-1}$. This relation is approximately correct for a sublimation temperature of $1500 \mathrm{~K}$ of graphite grains with an average radial size of $0.05 \mu \mathrm{m}$ (e.g., Barvainis 1987; Kishimoto et al. 2007; also assuming that the bulk of the bolometric contribution stems from ultraviolet photons). All sources with $r_{0} / r_{\text {sub }}$ less than this threshold were classified as "well-resolved". In other words, the threshold is a simple multiple of the expected physical size of the torus, and the suggestion is that the rest of the "less-resolved" sources are likely to have non-negligible contamination from extended emission that falls within the central diffraction Airy ring on the VISIR images.

In this paper we use the same source classification criterion for consistency and discuss its limitations and the effects of different assumptions in Sect. 5. We thus find 22 (of the total 42) sources to be well-resolved, including all Compton-thick sources except NGC 5135. Performing a least-square fit on this sample with a simple power law (i.e. a linear relation of the form $\log L_{\mathrm{MIR}}=a+b \log L_{\mathrm{X}}$ in $\log$-space) gives the following best-fit correlation:

$\log \left(\frac{L_{\mathrm{MIR}}}{10^{43}}\right)=(0.19 \pm 0.05)+(1.11 \pm 0.07) \log \left(\frac{L_{\mathrm{X}}}{10^{43}}\right)$ where the luminosities are normalized to $10^{43} \mathrm{erg} \mathrm{s}^{-1}$, the approximate mean power of the sample. The regression accounts for luminosity errors on both axes and the uncertainties are based on $\Delta \chi^{2}=1$ for each best-fit parameter (cf. fitexy procedure in Press et al. 1992). This correlation curve is over-plotted in Fig. 1. Equation (2) is completely consistent with (but slightly tighter than) that derived from the smaller sample in Paper II. For easy comparison with that paper, we also state the correlation fit without any normalization, in units of $\mathrm{erg} \mathrm{s}^{-1}$ directly: $\log L_{\mathrm{MIR}}=(-4.37 \pm 3.08)+(1.106 \pm 0.071) \log L_{\mathrm{X}}$. The formal significance of the correlation, according to the Spearman Rank coefficient $(\rho)$, is $\rho=0.93$ with a standard Student- $t$ null significance level of $3 \times 10^{-10}$. As discussed in Paper II, the correlation is not just a consequence of correlated distances on both axes; an intrinsic flux-flux correlation is also present, with a slightly smaller $\rho=0.86$ at a null significance level of $3 \times 10^{-7}$. This can also be seen from a partial correlation analysis between $\log L_{\mathrm{X}}$ and $\log L_{\mathrm{MIR}}$, with the correlation component due to redshift removed. These values are listed in Table 2 . The table lists in the first three columns: Col. (1) various subsamples of interest; Col. (2) number of sources in each subsample $(N)$; Col. (3) partial correlation coefficient $\left(\rho_{\cdot z}\right)$ between the logarithmic luminosities.

When fitting the correlation to all 42 sources without any selection, the change in parameters is closely equivalent to a simple increase in the intercept on the axis of $\log L_{\text {MIR }}$ by a value of 0.24 (i.e., a mean $L_{\text {MIR }}$ larger by a factor of 1.7 , consistent with inclusion of more IR flux in the cores of the lessresolved subsample). In this case, $\rho=0.88$ at a null significance level of $1 \times 10^{-14}$. On the other hand, excluding the Comptonthick sources has an insignificant effect. A few representative fits to various subsample populations are also listed in Table 2; Col. (4) lists the regression intercept and its 1- $\sigma$ uncertainty, while Col. (5) shows the corresponding slope values.

\section{The intrinsic mid-IR emission of AGN cores}

\subsection{Dispersion in $L_{\mathrm{MIR}}$ vs. $L_{\mathrm{X}}$}

The measured $L_{\mathrm{MIR}}$ values correlate tightly with $L_{\mathrm{X}}$ over three orders of magnitude in Seyfert luminosity. The small scatter 


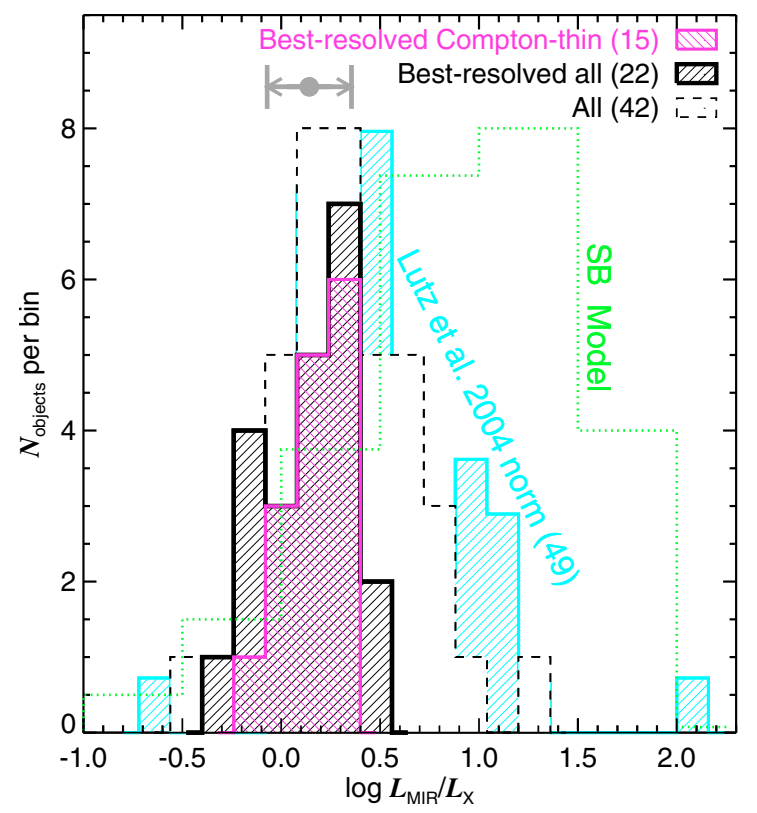

Fig. 2. Dispersion in $\log L_{\mathrm{MIR}} / L_{\mathrm{X}}$. For our data, three distributions are plotted in order of increasing depth into the figure: i) for 15 wellresolved Compton-thin sources is shown by the pink cross-hatched histogram; ii) for all 22 well-resolved sources by the black hatched area; and iii) for all 42 sources by the unfilled histogram with a dashed outline. The grey filled circle and arrow shows the mean and extreme values of the correlation curve in Eq. (2) over the fitted luminosity range. For the sample of Lutz et al. (2004), the turquoise hatched area shows the distribution of 49 sources in $\log \left(\lambda L_{\lambda}^{6 \mu \mathrm{m}} / L_{\mathrm{X}}\right)$, normalized to the maximum value of our full data histogram. Finally, the light-green, dotted histogram is the model prediction for the AGN+starburst model of Ballantyne (2008), also normalized.

is emphasized by plotting the distribution of the log-space luminosity ratio $r=\log \left(L_{\mathrm{MIR}} / L_{\mathrm{X}}\right)$ in Fig. 2. Our full dataset of 42 sources has a mean and standard deviation of $\left(\bar{r}, \sigma_{r}\right)=$ $(0.30,0.36)$ in this ratio, while the corresponding values for the 22 well-resolved sources of all types are $(0.15,0.23)$. The 15 Compton-thin well-resolved sources have the least scatter of all with corresponding values of $(0.17,0.18)$. This can be seen in the righthand plot in Fig. 1, and in Table 2 whose Cols. (6) and (7) list the ratio mean and standard deviation for various subsamples, respectively. The dispersion in $\log L_{\mathrm{MIR}}$ with respect to the best-fit correlation curves (i.e. observed vs. predicted $\left.\log L_{\mathrm{MIR}}\right)$ in the individual subsamples are very similar to the above $\sigma_{r}$ values.

The turquoise histogram in the same figure refers to the ISO study of Lutz et al. (2004), for which we present their distribution in $\log \left(\lambda L_{\lambda}^{6 \mu \mathrm{m}} / L_{\mathrm{X}}\right)$ of $49 \mathrm{AGN}$ - the ones with $6-\mu \mathrm{m}$ continuum detections for which intrinsic X-ray flux estimates could be determined. We also removed any sources likely to have significant IR starburst contamination (as defined by these authors). For this distribution, $\left(\bar{r}, \sigma_{r}\right)=(0.53,0.45)$. The spread is comparable to (only slightly larger than) the scatter for our full sample of 42 sources. The mean value, on the other hand, is significantly higher than for our sample, which may stem either from contamination within the larger ISO apertures or from the heterogeneous nature of their sample; we return to this in Sect. 4.6. We can at least confidently rule out this difference being caused by their bluer $6 \mu \mathrm{m}$ central wavelengths. Using median mid-IR AGN SEDs presented by Hao et al. (2007), we find that $\lambda L_{\lambda}^{6 \mu \mathrm{m}}$

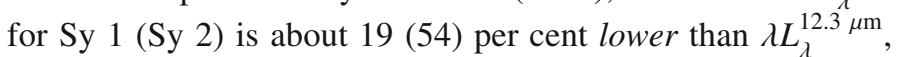

which is all due to red continuum shapes and not because of contamination by PAH emission or silicate absorption. Thus wavelength differences alone should have resulted in a lower mean $\log \left(\lambda L_{\lambda}^{6 \mu \mathrm{m}} / L_{\mathrm{X}}\right)$ than our $12.3 \mu \mathrm{m} \bar{r}$ values. If we do convert the Lutz et al. luminosities to rest-frame $\lambda L_{\lambda}^{12.3 \mu \mathrm{m}}$ using the Sy 1 , Sy 2 and quasar $k$-corrections from Hao et al., we find $\left(\bar{r}, \sigma_{r}\right)=$ $(0.71,0.50)$.

With regard to the well-resolved sources, is the small dispersion of this subsample caused by serendipitous selection of a few sources from our full sample which, in reality, have no physical interrelation? The dispersion of a smaller sample could turn out to be low due to statistical, rather than physical reasons, as pointed out by Lutz et al. (2004). We rule out this possibility based on two arguments. First, note the clear and large differences between the scatter of the well-resolved subsample and of other subsamples containing approximately equal objects in Table 2. The difference is especially clear when comparing against the less-resolved sources that have much higher $\left(\bar{r}, \sigma_{r}\right)=$ $(0.5,0.4)$ values. Next, to estimate the effect of any fortuitous selection further, we constructed 30000 bootstrap populations of 22 sources each, drawn randomly from the full sample of 42 sources without replacement. We measured the dispersion in each of these populations and computed the fraction of random populations with a dispersion less than or equal to the dispersion measured in the data. The result is that our measured data dispersion is robust to a serendipitous selection at 99.2 per cent; only about 250 random subsamples had a variance smaller than that of the data.

\subsection{The compositions of the unresolved mid-IR cores}

The imaged VISIR cores of the vast majority of our full sample appear completely unresolved, even at the high resolution of the VLT (as mentioned above; see also extensive discussion in Paper III). The physical resolution limits $\left(r_{0}\right)$ of our observations range over tens to hundreds of pc (see Table 1; Paper II). This is higher by factors of several hundred than an inner torus radius computed according to Eq. (1). The full radial extent of the torus, on the other hand, is more difficult to constrain (e.g. Granato et al. 1997). But our resolution limit is still larger by factors of a few to several tens than the outer radii suggested by recent clumpy torus models (cf. Hönig et al. 2006; Nenkova et al. 2008b, and references therein). Our listed $r_{0}$ values should thus be taken as strong upper limits of the physical sizes of the individual tori (at least the size relevant for the distribution of hot mid-IR-emitting dust).

What about the composition of the unresolved AGN cores? The small scatter in our correlation can be used to constrain their average composition to some extent because any contribution by multiple, unresolved components is bound to increase the dispersion in $\log \left(L_{\mathrm{IR}} / L_{\mathrm{X}}\right)$.

A very tight correlation may argue for a single population of particles emitting both X-ray and IR photons via some nonthermal process. Though we cannot discount an important nonthermal mid-IR component for every one of our sources, such a component is not the dominant cause of the tight correlation, as can be argued in several ways. Only a few of our targets show significant nonthermal emission in the radio, including 3C 445 and Cen A (in which a nonthermal mid-IR synchrotron component has indeed been inferred to exist by Meisenheimer et al. 2007; though see Radomski et al. 2008, for other possibilities). Furthermore, the X-ray power laws, if extended to the IR regime, severely underestimate the observed VISIR fluxes in most cases 
(but see Carleton et al. 1987). Finally, extensive modeling of individual sources also supports the weakness of any nonthermal component. An extended mid-IR structure has been resolved out with interferometric observations (Jaffe et al. 2004) in at least one of the objects, NGC 1068. Hönig et al. (2008a) have modeled the broad-band emission from this source and placed strict limits on the nonthermal radiation, favoring instead thermal emission from a clumpy torus cloud medium.

In any case, our empirical luminosity correlation is valid and important, regardless of its underlying physical origin. We leave further discussion of this issue to future works, including detailed monitoring observations and modeling of AGN tori; instead, we focus now on another consequence of the small correlation spread - limits on the spatial extent and power of star formation (SF) in the mid-IR at high spatial resolution.

\subsection{Constraining unresolved mid-IR star formation}

Powerful nuclear starbursts (SB) should produce copious amounts of infrared emission, but comparatively little X-rays (e.g. Rodriguez Espinosa et al. 1987; Ranalli et al. 2003). While the bulk of this is radiated at far-IR wavelengths (at $\gtrsim 60-\mu \mathrm{m}$ ), the mid-IR flux around $10-\mu \mathrm{m}$ due to SF is far from negligible (e.g. Maiolino et al. 1995; Galliano et al. 2005a; Siebenmorgen et al. 2008; our Papers II and III). Furthermore, the instantaneous IR luminosity of an SB can vary by more than an order of magnitude (e.g. Cid Fernandes et al. 2004; Davies et al. 2007), depending on its size and age. The resulting expected scatter in the combined AGN+SB power can be large. On the other hand, our tight mid-IR:X-ray correlation suggests that AGN emission in the point cores of our high-resolution imaging completely dominates any SF on similar scales at mid-IR wavelengths, and provides us the opportunity to place limits on any unresolved SB contribution.

In a recent work, Ballantyne (2008) investigates the efficacy of nuclear SBs at $z \sim 1$ for obscuring the sources that power the bulk of the cosmic X-ray background radiation. Although the physical motive and cosmic epoch of Ballantyne's study is different from ours, his numerical models make useful predictions of the emitted mid-IR (from the SB) and X-ray (AGN) fluxes, which can be compared with our observations. In particular, the expected distribution of $\log \left(\lambda L_{\lambda}^{6 \mu \mathrm{m}} / L_{\mathrm{X}}\right)$ from his Fig. 11 is illustrated with the green histogram in Fig. 2. For this modeled distribution, $\sigma_{r} \approx 0.75$, a factor of about 3 (2) greater than our sample of well-resolved (all) sources, though it must be noted that the nuclear SB luminosity ratios are only indicative values, according to Ballantyne. Conversion of the model luminosities to $12 \mu \mathrm{m}$ should not affect the dispersion much (cf. Fig. 7 in Ballantyne 2008, in which the 6 and $12 \mu \mathrm{m}$ SB luminosities are approximately equal).

Star formation is certainly present around the cores of Seyferts, but it is the spatial scale being probed that is the important quantity here. As an illustrative example of this, in Fig. 3 we show our VISIR high-resolution imaging of one target IC 3639 - whose nuclear SB was studied in detail by González Delgado et al. (1998) and found to have a bolometric luminosity (determined mainly from ultraviolet observations) very similar to the total power emitted by the central AGN. The point-like mid-IR profile sharply contrasts to the over-plotted contours of HST imaging obtained by these authors. Images of all our targets from Papers I and II can be found in Paper III (Horst et al. 2009); other multi-band images and SEDs from the present sample will be presented in a future work.

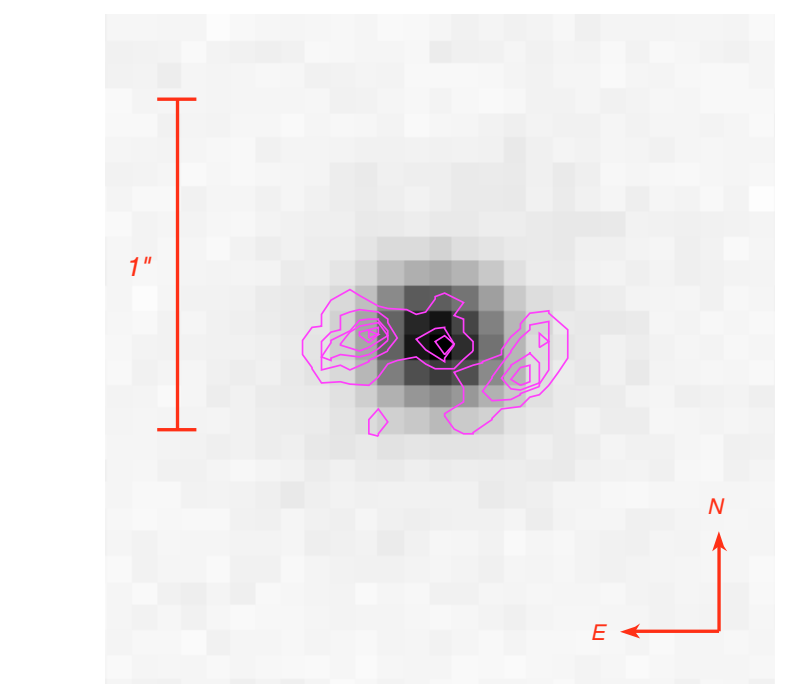

Fig. 3. VISIR NEII_2 $\left(\lambda_{\mathrm{C}}=13.04-\mu \mathrm{m}\right)$ image of IC 3639, showing the clean point-like core profile. North is up and east to the left; the image was obtained at 0 ! $^{\prime} 075 /$ pixel sampling and is $2^{\prime \prime} \times 2$ " wide. The overlaid contours are from the ultraviolet (UV) Faint Object Camera image obtained from the HST archive. Contour levels range from $10 \%$ to $100 \%$ of the peak UV flux. The extended SB emission over the central arcsec, especially the central "horizontal-S" shaped structure of knots (cf. González Delgado et al. 1998) are clearly visible in the UV, but not at $12-\mu \mathrm{m}$. (This figure is available in color in electronic form.)

The median resolved scale $\left(r_{0}\right)$ for our 22 targets classified as being well-resolved is $70 \pm 11 \mathrm{pc}$, and for the rest of the 20 lessresolved targets is $115 \pm 24 \mathrm{pc}$ (errors correspond to the standard deviation of the median of 10000 bootstrap samples drawn with replacement). Another way to gauge the difference between the two samples is a two-sided Kolmogorov-Smirnov (KS) test, according to which the distributions of resolved scales differ at the 97 per cent level. On the other hand, the distributions of $r_{\text {sub }}$ values of the two samples are consistent with each other. In other words, the dominant factor governing source classification turns out to be simply the distance, with the well-resolved sources being on average closer to us (their median redshift is 0.010 , while $z_{\text {median }}$ for the rest is 0.017 ) and with lower $r_{0}$ values.

It then follows that the relevant scale within which the AGN completely dominates the SB is the central $\sim 60-80 \mathrm{pc}$ (the median of the well-resolved sample) of the nuclei of local Seyferts. In Table 2, we found $\sigma_{r}=0.23$ for the well-resolved sample. If this scatter were to be solely due to nuclear SB and assuming negligible X-rays from them, it would imply that SB within these physical scales typically have a $12 \mu \mathrm{m}$ luminosity of no more than $1-10^{-\sigma_{r}}=40 \%$ of the total unresolved (AGN+SB) $L_{\mathrm{MIR}}$. Assuming the dispersion for the full sample of 42 sources increases this limit to $56 \%$ while the use of the Compton-thin subsample reduces it to $34 \%$. These are conservative upper-limits, because other effects such as AGN infrared variability and the scatter due to statistically-differing distributions of torus clouds will introduce additional uncertainty, so the true constraint will undoubtedly be even tighter.

Beyond a radius of $\sim 100 \mathrm{pc}$ (using the less-resolved sample alone), the limit on the SB power increases to $\sim 1.5$ times the $12 \mu \mathrm{m}$ luminosity of the AGN itself, allowing for the bulk of star formation to occur on these scales. This is completely consistent with other recent studies: e.g. Imanishi (2002), Soifer et al. (2003), and Davies et al. (2007). The last authors obtained detailed integral-field near-IR spectroscopy of several local Seyferts and found AGN and nuclear SB powers to only 
be comparable on kpc scales. $K$-corrections of their results to $12-\mu \mathrm{m}$ are expected to be small compared to the differences due to integration on different spatial scales. Finally, we emphasize that, although our observed scatter is much smaller than that presented by Ballantyne (2008) this is not, by any means, a negation of their model, which is relevant at $z \sim 1$. It simply means that we are resolving any similar SBs at the present epoch, if they exist.

In summary, we suggest that our high-resolution observations are predominantly probing the nuclear torus emission, unbiased by any overwhelming contamination due to powerful SF.

\subsection{Infrared:X-ray spectral indices}

Having determined the true mid-IR and X-ray luminosities attributable to the AGN themselves, we can now derive their intrinsic SED shapes. A spectral index connecting two widelyseparated frequencies is often used to describe sparsely sampled broad-band spectra. In accordance with the widely-used optical:X-ray spectral index $\left(\alpha_{\mathrm{OX}}\right)$, we define the index

$\alpha_{\mathrm{IX}}=\frac{\log \left(L_{2 \mathrm{keV}}^{v} / L_{12.3 \mu \mathrm{m}}^{v}\right)}{\log \left(v_{2 \mathrm{keV}} / v_{12.3 \mu \mathrm{m}}\right)}=0.233 \log \left(L_{2 \mathrm{keV}}^{v} / L_{12.3 \mu \mathrm{m}}^{v}\right)$

where $L^{v}$ represent the monochromatic luminosities [erg s $\mathrm{Hz}^{-1}$ ] at frequencies $(v)$ corresponding to $2 \mathrm{keV}$ and $12.3 \mu \mathrm{m}$, both in the rest-frame. We computed $L_{2}^{v} \mathrm{keV}$ from the individual $\mathrm{X}$-ray photon-indices (usually determined from spectral fits over the $\sim 0.5-12 \mathrm{keV}$ range) in the literature. The $\alpha_{\text {IX }}$ values for each source are listed in Table B.1.

We then find a mean $\alpha_{\mathrm{IX}}=-1.10 \pm 0.01(0.05)$ and $-1.18 \pm$ $0.02(0.09)$ for the well-resolved and less-resolved samples, respectively, and $-1.14 \pm 0.01(0.08)$ for the whole sample; the numbers in brackets list the full scatter $\left(\sigma_{\alpha_{\mathrm{IX}}}\right)$. This distribution is shown in Fig. 4. The plot shows that not only is the spectral slope for the less-resolved sources steeper (consistent with including more mid-IR emission for the same $L_{X}$ ), but the dispersion of slopes for these sources is also greater.

Often in deep surveys, it is not possible to determine the $\mathrm{X}$-ray photon-index from individual spectral fits, because of the faintness of sources. For such cases, we note that it a good approximation to assume a uniform $\Gamma \approx 1.9$ over the $2-10 \mathrm{keV}$ range. This is because the typical observed range of $\Gamma$ is $\approx 1.5-2.3$ (e.g. Nandra \& Pounds 1994; Mateos et al. 2005; Piconcelli et al. 2005), and the difference between a constant $\Gamma=1.9$ and the extremes of the stated range results in only a small difference of $\Delta \alpha_{\mathrm{IX}}= \pm 0.04$ (assuming no change in $L_{\mathrm{X}}$ ). In terms of predicting the luminosity of a source at one frequency from a flux (i.e. luminosity) measurement at the other frequency, using the average spectral slope computed above will give an answer that is accurate to within a factor of 1.5 , over the mentioned range of X-ray photon-indices.

It is well-known that the ultraviolet:X-ray spectral index $\left(\alpha_{\mathrm{OX}}\right)$ of AGN is strongly dependent upon luminosity (e.g. Vignali et al. 2003; Steffen et al. 2006). The correlation of Eq. (2) is only slightly nonlinear, which implies that the corresponding mid-IR:X-ray index should instead depend only weakly on luminosity. Assuming again that a constant X-ray photon-index $(\Gamma=1.9)$ is applicable to all sources for simplicity, the expected power law index of dependence of $\alpha_{\mathrm{IX}}$ on the monochromatic IR luminosity is easily derived from Eqs. (2) and (3) to be $0.233\left(b^{-1}-1\right)$, where $b$ is the best-fit correlation slope. We found $b=+1.11$ for the well-resolved subsample, which implies an expected dependence of $\alpha_{\mathrm{IX}} \propto\left(L_{\mathrm{MIR}}^{v}\right)^{-0.02}$. The righthand panel of
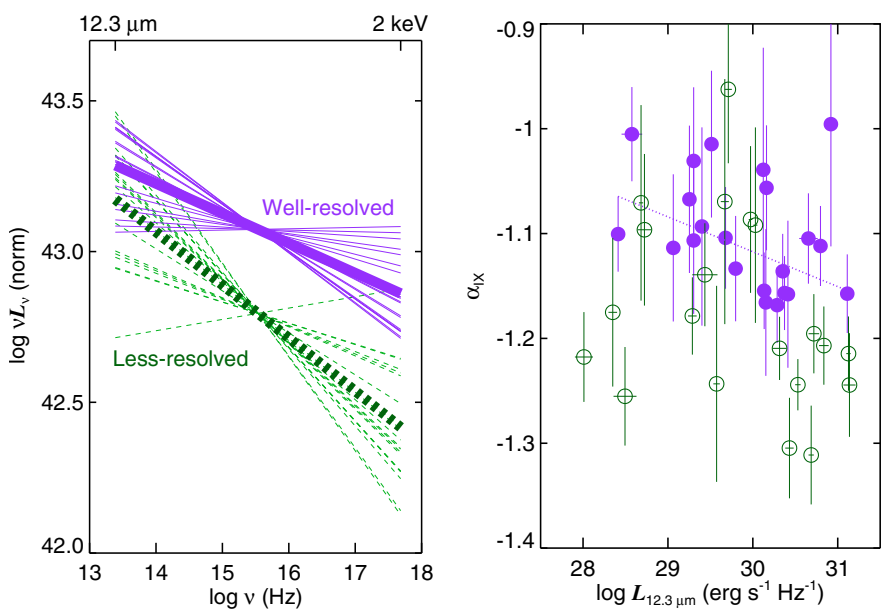

Fig. 4. Left: the two-point broad-band SEDs of the well-resolved (purple, solid lines) and less-resolved (green, dashed lines) subsamples, showing the distribution of $\alpha_{\mathrm{IX}}$ values. Within both subsamples, sources have been normalized for clarity to the average subsample luminosity at the mean frequency of $\log v=15.54$. The thick central lines denote the mean $\alpha_{\mathrm{IX}}$ slopes. Right: the distribution of individual $\alpha_{\mathrm{IX}}$ values as a function of $\log L_{12.3 \mu \mathrm{m}}^{v}$. The dotted line is the best-fit regression curve for the well-resolved subsample and it has a slope close to the expected value of -0.02 . (This figure is available in color in electronic form.)

Fig. 4 shows the full dataset of individual sources in the $\alpha_{\mathrm{IX}}$-vs.$L_{\mathrm{MIR}}^{v}$ plane, and the errors on $\alpha_{\mathrm{IX}}$ incorporate the uncertainties on both X-ray and mid-IR luminosities. The regression of the well-resolved sources, and its best-fit slope of $-0.033 \pm 0.013$, is consistent with the expected value.

\subsection{Bolometric corrections}

Correction factors $\left(f_{\mathrm{Bol}}\right)$ for obtaining full bolometric AGN power emissions based on observations over limited energy ranges are crucial for computing the total accretion energy release in the cosmos, as well as studying black hole growth and evolution (e.g. Elvis et al. 1994; Fabian \& Iwasawa 1999; Marconi et al. 2004; Hopkins et al. 2007; Gilli et al. 2007). In particular, it is natural to connect source distributions seen at mid-IR and X-ray energies (e.g. Gandhi \& Fabian 2003) as these are also good probes of any evolution in obscured AGN populations (e.g. Ballantyne \& Papovich 2007; Treister et al. 2008).

Intrinsic bolometric corrections connecting the absorptioncorrected X-ray power with the integrated (optical/ultraviolet to hard X-ray) emission have been found to increase with AGN luminosity (e.g. Marconi et al. 2004; see also above discussion on $\left.\alpha_{\mathrm{OX}}\right)$. If the mid-infrared flux, on the other hand, is the result of reprocessing and thermalization of intrinsic AGN photons within torus dust clouds, then the infrared correction factor will depend upon radiative transfer within each cloud, as well as the clouds covering factor.

From an empirical standpoint, the expected infrared bolometric corrections $\left(f_{\mathrm{Bol}}^{\mathrm{MIR}}=L_{\mathrm{Bol}} / L_{\mathrm{MIR}}\right)$ can be easily derived by combining our $L_{\mathrm{MIR}}: L_{\mathrm{X}}$ correlation with the $L_{\mathrm{Bol}}: L_{2-10}$ trend found by Marconi et al. (2004). These authors present the X-ray bolometric corrections $\left(f_{\mathrm{Bol}}^{\mathrm{X}}=L_{\mathrm{Bol}} / L_{2-10}\right)$ as a function of the scaled bolometric luminosity $\mathcal{L}=\log \left(L_{\mathrm{Bol}} / L_{\odot}\right)-12$, and we adopt the same units to ease comparison. The value of $L_{\odot}$ used is $3.826 \times 10^{33} \mathrm{erg} \mathrm{s}^{-1}$. The following equation is obtained:

$\log f_{\mathrm{Bol}}^{\mathrm{MIR}}(\mathcal{L})=b \log f_{\mathrm{Bol}}^{\mathrm{X}}(\mathcal{L})-a+(1-b)\left(\mathcal{L}+12+\log L_{\odot}-43\right) .(4)$ 


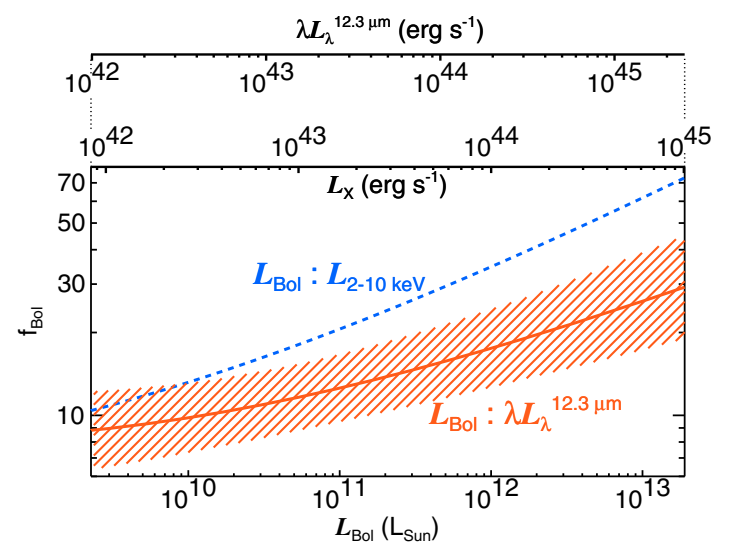

Fig. 5. Mid-infrared bolometric correction $f_{\mathrm{Bol}}^{\mathrm{MIR}}=L_{\mathrm{Bol}} / L_{\mathrm{MIR}}$ (red continuous curve) - determined from our $L_{\mathrm{MIR}}: L_{\mathrm{X}}$ correlation for wellresolved sources (Eq. (2)) and the $f_{\mathrm{Bol}}^{\mathrm{X}}=L_{\mathrm{Bol}} / L_{\mathrm{X}}$ trend observed by Marconi et al. (2004). This X-ray trend is shown as the blue dashed curve. The red hatched area shows the 16th and 84th percentile confidence region in 10000 Monte Carlo randomizations, incorporating normal uncertainties on our correlation parameters, as well as on $\log f_{\mathrm{Bol}}^{\mathrm{X}}$. (This figure is available in color in electronic form.)

Expressing it in this form makes clear the dependence on our fitted slope and intercept (which can be picked from Table 2 for any of the fits), and the additional log-luminosity units collected at the end. It also aids in Monte Carlo determination of confidence intervals, because Marconi et al. (2004) state an uncertainty of $0.1 \mathrm{in} \log f_{\mathrm{Bol}}^{\mathrm{X}}$, and we have computed the uncertainties in $a$ and $b$ in Table 2. Assuming these to be normally-distributed, the resulting 1- $\sigma$ uncertainty can be derived from the scatter in a large number (we used 10000) of randomizations of Eq. (4) at various values of $\mathcal{L}$. Substituting the best-fit $a$ and $b$ for our well-resolved sample (Eq. (2)), and expanding $\log f_{\mathrm{Bol}}^{\mathrm{X}}$ from the relevant Eq. (21) in Marconi et al. (2004) then gives

$$
\log f_{\mathrm{Bol}}^{\mathrm{MIR}}(\mathcal{L})=1.24+0.16 \mathcal{L}+0.012 \mathcal{L}^{2}-0.0015 \mathcal{L}^{3}
$$

This curve is drawn in Fig. 5, along with the computed uncertainty. The plot shows a monotonically-increasing $f_{\mathrm{Bol}}^{\mathrm{MIR}}$ correction for the Sy luminosities that we probe, with a range of average values of $\approx 10-30$, while the $\mathrm{X}$-ray correction is steeper.

It is important to note a caveat implied by more recent studies. Vasudevan \& Fabian $(2007,2009)$ find only a marginal trend of $f_{\mathrm{Bol}}$ with $L_{\mathrm{X}}$, inferring instead a stronger dependence on the the Eddington ratio that is proportional to $L_{\mathrm{Bol}}$ normalized to the black hole mass. In this case, infrared bolometric corrections may be expected to vary more weakly with luminosity than in Fig. 5, or to show more complex behavior.

\subsection{From Seyferts to quasars?}

Our study has probed local AGN, which are predominantly Seyferts. AGN with quasar-like X-ray luminosities $\left(L_{X} \gg\right.$ $10^{44} \mathrm{erg} \mathrm{s}^{-1}$ ) are absent from our sample due to this selection of nearby targets and because distant sources are less resolved. On the other hand, it becomes increasingly difficult for SF to dominate the energetics of sources at the high end of bolometric luminosities. The bulk of the observed IR fluxes of the most luminous Seyferts and quasars are likely to be generated by accretion onto the central black hole, irrespective of instrumental angular resolution. Thus, we should be able to check our correlation in the luminous quasar regime by using space-telescope observations of distant quasars.

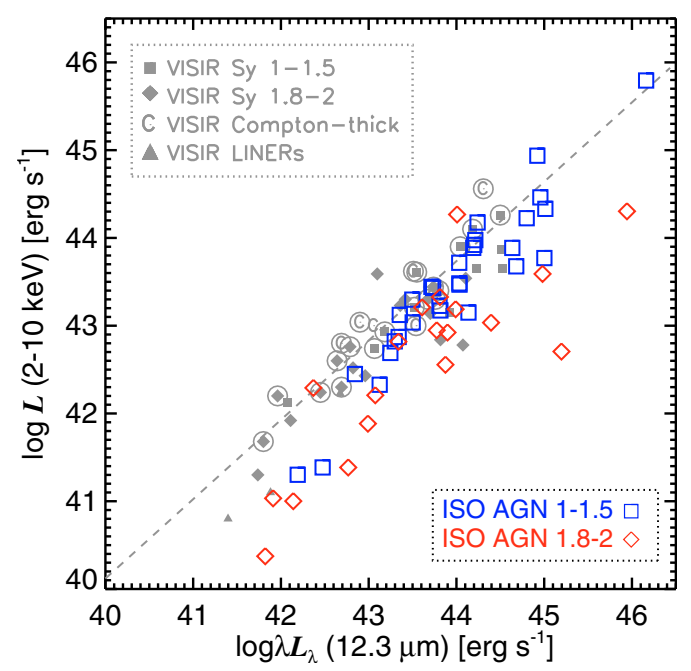

Fig. 6. Our correlation from Fig. 1, compared with the distribution of sources from Lutz et al. (2004, cf. their Fig. 5). Their $6 \mu \mathrm{m}$ continuum luminosities have been converted to $12.3 \mu \mathrm{m}$ using median $k$-corrections from Hao et al. (2007). Luminous unobscured AGN (1-1.5; blue empty squares; those least biased by contamination) fall smoothly onto our correlation in the quasar regime.

We tested this on the sample of Lutz et al. (2004), for which the authors had carefully decomposed AGN and host galaxy contributions using ISO spectra. The sample includes Seyferts, quasars, and ultra-luminous infrared galaxies (ULIRGs), and extends out to $z \approx 1$. We converted the $6 \mu \mathrm{m}$ continuum luminosities of the 49 sources used in Fig. 2 to $12.3 \mu \mathrm{m}$ with the $k$-corrections of Hao et al. (2007) for Sy 1 s and Sy 2s below $L_{\mathrm{X}}=10^{44} \mathrm{erg} \mathrm{s}^{-1}$ and the corresponding quasar SEDs above this limit, but we note that the conclusions below do not depend upon these corrections. The result is shown in Fig. 6.

It is seen that the ISO sample deviates significantly from our best-fit correlation at low luminosities but not at high luminosities, just as might be expected from contamination at low powers. The match is particularly good for the brightest unobscured quasars (with $\log L_{X} \gtrsim 44$ ), whose observed emission should be the least biased by any extended SF.

The deviations of the type $2 \mathrm{AGN}$, on the other hand, are large even for luminous objects. The sense of the deviation is such that a higher IR power is observed at any given $L_{X}$, compared to the distribution of type 1 AGN. Examining the three most luminous type 2 AGN, we find that all of them are actually ULIRGs: IRAS 23060+0505 (Brandt et al. 1997), IRAS 05189-2524 (Severgnini et al. 2001), and Mrk 463 (Bianchi et al. 2008). The observed core structure of ULIRGs is quite different from Seyferts and quasars. Many ULIRGs are seen to occur in strongly interacting and merging systems where gas gets channeled to the cores and drives very compact and powerful SBs. Two X-ray emitting AGN are sometimes seen in close proximity - one from each of the merging systems. Therefore, no apriori match of ULIRGs (i.e., without extensive corrections) is expected with our Sy mid-IR:X-ray correlation. Furthermore, the X-ray luminosity of at least one of these Mrk 463 - has been revised upwards by new Chandra imaging (see Bianchi et al. 2008, who find a binary AGN with higher $L_{X}$ than previous estimates), thus actually moving it closer to our correlation line.

With all these caveats in mind, we regard the match of the luminous sources from Lutz et al. (2004, at least the unobscured ones) to be fair; consequently, we suggest that Eq. (2) can be extended into the quasar regime as well. Interestingly, our 
correlation is straddled by the two correlation slopes quoted by Fiore et al. (2009, see their Eq. (1)) for Sy and quasars respectively in the CDF-S and C-COSMOS fields. Of course, further detailed validation with enlarged samples will be important.

\section{Systematic effects}

\subsection{To be or not to be well-resolved}

As described in Sect. 3, our threshold for classifying sources as well-resolved or not is based on locating a discontinuity in the $L_{\mathrm{MIR}} / L_{\mathrm{X}}$-vs.- $r_{0} / r_{\text {sub }}$ (luminosity ratio vs. normalized scale) parameter space. The reasoning is that, for sources at the higher end of the distribution of VISIR core physical size scales (as compared to the torus), significant unresolved contamination is allowed and can lead to an increase in the scatter, as well as the mean $L_{\mathrm{MIR}}$ at any given X-ray power. These sources are the "less-resolved" ones, the rest being "well-resolved". Because such an empirical search has important limitations, we need to discuss these in some detail. But we emphasize that the correlation for all 42 sources remains robust (with a small scatter relative to previous studies) even if no selection based on resolved scale is applied (see Fig. 2).

First, adding the targets from this paper to the luminosityratio vs. normalized scale plot of Paper II (Fig. 3 of that work), the full new distribution is plotted in the lefthand part of Fig. 7. A crude uncertainty in the normalized scale threshold can be estimated simply from the mean errors of the binned source distribution around the adopted threshold of $r_{0} / r_{\text {sub }}=560$, which suggests $\Delta\left(r_{0} / r_{\text {sub }}\right) \approx 40$. Thus increasing (decreasing) our threshold to $r_{0} / r_{\text {sub }}=600(520)$, the number of well-resolved sources changes from 22 to 25 (16). The corresponding bestfit slope steepens (flattens) to 1.21 (1.07). The details of these fits are listed in Table 2 as "Well-resolved (Systematic 1 $\uparrow$ )" and "(Systematic $1 \downarrow)$ " respectively.

Second, in Paper II, a linear scaling of $L_{\mathrm{Bol}}=10 L_{\mathrm{X}}$ was assumed in the computation of $r_{\text {sub }}$. On the other hand, bolometric corrections may increase with source luminosity (as discussed in the previous section), in which case $r_{\text {sub }}$ will increase faster than implied by the linear dependence above, and will result in more of the luminous sources satisfying any given threshold in $r_{0} / r_{\text {sub }}$. Using the $f_{\mathrm{Bol}}-L_{\mathrm{X}}$ relation of Marconi et al. (2004), we recomputed $r_{\text {sub }}$ (and hence the normalized scale $r_{0} / r_{\text {sub }}$ ) as a function of $\log \left(L_{\mathrm{MIR}} / L_{\mathrm{X}}\right)$, and the resulting distribution is shown in the righthand panel of Fig. 7. On average, the normalized scales are shifted to lower values due to the faster increase in $r_{\text {sub. }}$. In order to best determine the scale where the average distribution of $\log \left(L_{\mathrm{MIR}} / L_{\mathrm{X}}\right)$ undergoes a change, we adopted a floating threshold in normalized scale. The distributions above and below this floating threshold were compared with a two-sided KS test. A value of $r_{0} / r_{\text {sub }}=420$ proved to be the best discriminator, with the two distributions (well-resolved vs. less-resolved) differing at 98.6 per cent. This can also be roughly discerned by eye in the plot as the noticeable change in the binned distribution, and the mean and scatter of the resulting well-resolved and less-resolved subsamples are $\left(\bar{r}, \sigma_{r}\right)=(0.16,0.27)$ and $(0.45$, $0.38)$, respectively. The number of sources that satisfy this wellresolved threshold is 22 (a slightly different set of 22 from that discussed in all previous sections) and the resulting $L_{\mathrm{MIR}}-L_{\mathrm{X}}$ correlation fit is largely unchanged; see details in Table 2 under heading "Well-resolved (Systematic 2)". The correlation parameters are thus insensitive to changes in the resolution threshold, as long as the corresponding well-resolved subsample is selfconsistently defined.

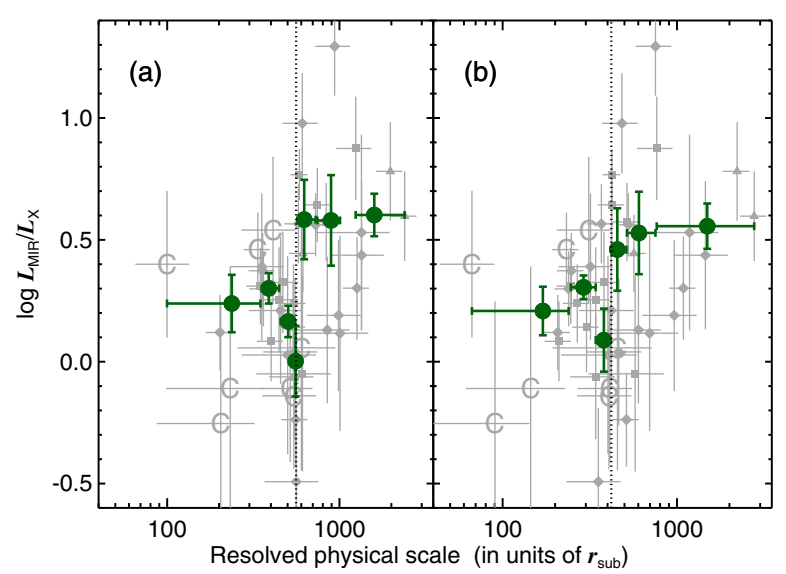

Fig. 7. Left a): logarithmic luminosity-ratio vs. normalized resolved scale $\left(r_{0} / r_{\text {sub }}\right)$, with $r_{\text {sub }}$ determined according to a constant linear scaling of $L_{\mathrm{Bol}}$-vs. $-L_{\mathrm{X}}$. The primary adopted threshold of $r_{0} / r_{\mathrm{sub}}=560$ is shown as the dotted vertical line. Sources falling to the left of this line are taken to be "well-resolved", and the rest "less-resolved". All 42 sources with symbols as in Fig. 1 are shown in grey, with the errors on scale accounting for uncertainty in $L_{X}$ and slight variations in midIR seeing. Bins in $r_{0} / r_{\text {sub }}$ are shown by the dark-green bold circles with horizontal error-bars denoting full bin widths. The standard deviation of the mean log-luminosity ratio in each bin is shown by the vertical error-bars on these circles. Right b): in this plot, $L_{\mathrm{Bol}}$ (and hence $r_{\mathrm{sub}}$ ) is determined according to the bolometric corrections of Marconi et al. (2004). The threshold is the best-fit discriminatory scale at $r_{0} / r_{\text {sub }}=420$. See Sect. 5.1. (This figure is available in color in electronic form.)

Finally, we note that, while recent work has confirmed the dependence of $r_{\text {sub }} \propto \sqrt{L}$ used in Eq. (1) (Suganuma et al. 2006), the absolute normalizing scale (in pc) of the relationship remains uncertain. Kishimoto et al. (2007) have shown that the innermost torus radii inferred from near-IR reverberation mapping of type 1 AGN are systematically smaller by a factor of $\sim 3$ than would be implied by the widely-used canonical assumptions implicit in Eq. (1). They discuss various alternatives to account for this, including a sublimation temperature higher than used for graphite $(\sim 1500 \mathrm{~K})$, or a typical grain size of $\sim 2 \mu \mathrm{m}$ - about four times larger than usually quoted. In either case, a smaller $r_{\text {sub }}$ will result in a simple translation of all our sources to higher $\mathrm{x}$-axis $\left(r_{0} / r_{\text {sub }}\right)$ values in Fig. 7 . The large change in $\log \left(L_{\mathrm{MIR}} / L_{\mathrm{X}}\right)$ will then be seen to occur around $1700 \times r_{0} / r_{\text {sub }}$ (using the factor $\sim 3$ offset found by Kishimoto et al.). Again, if the threshold is consistently chosen, there is no effect on our correlation.

\subsection{Treatment of errors}

The individual X-ray error bars are a significant source of uncertainty for the correlation. Our strategy has been to prefer published analyses with broad-band spectral modeling and to assign an error based on the range of source variability and fit parameters inferred in the recent literature. If the IR indeed originates from reprocessing in the torus, then the relevant quantities to compare are not necessarily X-ray observations obtained simultaneously with the IR, but rather the long-term (few years; relevant for a pc-scale torus) X-ray average flux. The IR flux, on the other hand, is expected to be much less variable. The range of published fits spanning various missions and several years (in addition to spectral complexities) results in much larger X-ray error bars than the corresponding IR ones. 
For simplicity, we adopted equal-length (high and low) error bars in log-luminosity space, which should be approximately correct given the many sources of uncertainty. On the other hand, it may be more natural to use equal-length error bars in linear space because flux (and count) measurement errors are usually normally distributed. Thus directly fitting a power law of the form $L_{\mathrm{MIR}}=a L_{\mathrm{X}}^{b}$ to the base sample of 22 well-resolved sources and accounting for the equal-length X-ray errors in luminosity, still results in a slope $b=1.11 \pm 0.10$, i.e. very similar to that of Eq. (2); see Table 2 "Systematic 3".

Finally, one may question the veracity of the statistical nature of our errors and ask what happens if we ignore these completely. We have tried a fit according to the algorithm of Isobe et al. (1990), which can be used when the error assignment and the nature of scatter about a linear relation are ill-understood. We directly used the log luminosities from the last two columns of Table 1 (and corresponding ones from Paper II) without the tabulated errors. Adopting the ordinary least-square bisector estimate recommended by Isobe et al. results in a regression slope of $b=1.02 \pm 0.07$, i.e. flatter than previous values and consistent with unity at $1-\sigma$ confidence. This procedure effectively gives identical weighting to all sources, including highly obscured ones, but it is precisely these last sources that tend to have larger inherent uncertainties (e.g. when correcting for Comptonthick obscuration) and that tend to lie above the correlation line in Fig. 1 (see discussion that follows in Sects. 5.4 and 6). We thus caution that such a fit may not be wholly appropriate, though we include it for completeness. The fitted slope and intercept are listed in Table 2 under "Systematic 4".

\subsection{Filter selection}

Depending on the redshift of each source, we have tried to choose narrow filters with central wavelengths that ought to be dominated by continuum emission. But we cannot rule out contamination due to emission line wings in every case. For instance, using the median Sy 2 SED from Hao et al. (2007), we estimate a worst-case contamination of the [NeII] $\lambda 12.81$ emission line in our NEII_2 filter photometry of about 14 per cent for the observation of NGC 3281.

As mentioned, we observed most sources in a number of filters around $12-\mu \mathrm{m}$. So we can gauge any overall bias on the correlation fit from selecting our particular filters by choosing instead the second most-appropriate filter from our set, i.e. the one whose central wavelength is closest to $12.3-\mu \mathrm{m}$, following the primary filter. These filters, and the related fluxes for the new targets in this paper, are listed in Table 1 as "secondary" filters. Including data from Papers I and II, we had access to 34 secondary filters fluxes out of the 42 sources; for the rest, we retain the primary fluxes. Converting these fluxes to $12.3-\mu \mathrm{m}$ luminosities as before, and fitting the new $L_{\mathrm{MIR}}-L_{\mathrm{X}}$ correlation reassuringly results in a negligible change in the correlation-fit parameters. Details are listed in Table 2 under the rows headed "Systematic 5". The dispersion in the correlation $\left(\sigma_{r}\right)$, on the other hand, does exhibit an increase. This is probably a consequence of the extra contamination in some of the bluer and broader secondary filters (i.e. SIV, SIC, PAH1; with central wavelengths around $\sim 9-12 \mu \mathrm{m}$ ) due to silicate absorption, $\mathrm{PAH}$, and other features.

In summary, the considerations of the three preceding sections suggest systematic variations in the mid-IR:X-ray correlation slope over $\sim 1.02-1.21$, in addition to statistical fit uncertainties.

\subsection{Infrared extinction corrections?}

No extinction corrections have been applied to the observed infrared fluxes in any of the above analysis. If, indeed, the tori in these sources are described better by high factors of clumpiness (e.g., Hönig et al. 2006) with discrete co-spatial clouds being responsible for both emission and obscuration, then such corrections will be invalid. As such, our tight correlation in Fig. 1 using uncorrected IR fluxes seems to be an aposteriori fact supporting (but not proving) the non-applicability of extinction corrections. Some previous studies have applied extinction corrections (Krabbe et al. 2001), while others have not (Lutz et al. 2004). In this section, we comment on the effect that canonical corrections would have on the inferred luminosities and the $L_{\mathrm{IR}}: L_{\mathrm{X}}$ correlation.

For our entire sample, the median $\log N_{\mathrm{H}} \sim 23.1$. Using the standard interstellar extinction law (Rieke \& Lebofsky 1985), the extinction $A_{12} \mu \mathrm{m}=0.03 A_{\mathrm{V}}$. A typical Galactic gas:dust ratio (Bohlin et al. 1978) then implies a median increase in the $L_{\mathrm{MIR}}$ values by a factor of 7 for our sample, if one were to correct the mid-IR fluxes for such reddening. On the other hand, for sources with higher obscuration, this correction will overestimate the intrinsic power. For instance, the correction factor for a source obscured by a gas column of $N_{\mathrm{H}}=4.5 \times 10^{23} \mathrm{~cm}^{-2}$ (the median value of our obscured sample) is about 1000 . A corresponding increase in $L_{\mathrm{MIR}}$ would end up pushing the bolometric luminosities well above the expected Eddington luminosities.

A possible solution may be to use smaller dust:gas ratios. Values of $A_{\mathrm{V}} / N_{\mathrm{H}}$ lower by a factor of $\sim 10$ than the Galactic value have been inferred for nearby Seyferts (Maiolino et al. 2001). This would moderate some of these large corrections, but would still overestimate the corrections for the extreme Compton-thick cases. We note that most of the sources with the lowest $L_{\mathrm{MIR}} / L_{\mathrm{X}}$ ratios (the ones highest above the correlation line in Fig. 1) are all highly obscured and one of these (NGC 7674) is Comptonthick. Recently, Zakamska et al. (2008) have found that deep Si absorption is more likely to occur in AGN with only the highest obscuration. The median $\mathrm{Si}$ optical depth for their likely Compton-thick sources is $\tau_{9.7} \approx 1.5$, but with a significant spread of $\tau_{9.7} \sim 1$ in their small sample (similar spreads have been inferred by Shi et al. 2006 and Hao et al. 2007). Assuming our highly-obscured sources also have similar IR opacities, and using a standard Milky Way opacity curve (Draine 2003) between 9.7 and $12-\mu \mathrm{m}$, correction factors of $\Delta \log L_{\mathrm{MIR}}=0.1-0.4$ (corresponding to $A_{\mathrm{V}}=7-35$ ) should be applied to obtain the intrinsic $12.3-\mu \mathrm{m}$ power of Compton-thick sources. Adopting such corrections has only a weak effect on the fitted correlation parameters in Table 2, changing them by less than their 1- $\sigma$ uncertainties.

To reiterate, we do not apply any IR extinction correction.

\section{Compton-thick sources}

Reliably identifying and gauging the intrinsic powers of Compton-thick (CT) AGN remains a difficult issue irrespective of source redshift (e.g. Comastri et al. 2007; Nandra \& Iwasawa 2007; Alexander et al. 2008). In most sources with extreme obscuring columns, indirect probes have to be employed and allowances made for extinction and contamination on extended scales around the nucleus (where the indirect flux emerges from). It may well be the case that both the X-ray and IR fluxes require additional corrections for obscuration and dust extinction, so there is no a priori reason to expect such sources to fit the correlation. 
It is therefore encouraging, if somewhat surprising, that our eight CT AGN with $\log N_{\mathrm{H}} \geq 24.2$ do not deviate significantly from the best-fit correlation. Because of the typically large uncertainties in their luminosities (see details in Appendix of this paper and of Paper II), CT AGN do not have any substantial effect on the correlation fit. But even ignoring these errors, the absolute scatter in $\log \left(L_{\mathrm{MIR}} / L_{\mathrm{X}}\right)$ is still moderate (see Fig. 2).

The importance of our study lies in how the mid-IR:X-ray correlation provides not only an independent, but also an accurate (with small scatter) way to probe AGN, regardless of any obscuration. Simple, high-resolution mid-IR imaging now provides a new proxy for AGN power including CT AGN. We can test this by searching for high-resolution mid-IR imaging observations of other CT Seyferts. Recently, Hönig et al. (2008b) obtained high spatial-resolution $N$-band spectroscopy with VISIR of a small sample of local AGN and discovered a strong mid-IR emission line Baldwin effect. Included in their sample are three CT AGN, of which NGC 1068 is already part of our sample. The second object, Circinus, is a special case (as discussed by these authors) and also showed significant extended emission; therefore, we do not consider it here. This leaves ESO 428-G014, which was first suggested as being CT from BeppoSAX observations by Maiolino et al. (1998). Hönig et al. (2008b) quote a $12-\mu \mathrm{m}$ flux of $0.22 \mathrm{Jy}=>\log L_{\mathrm{MIR}}=42.51$ at a distance of $22.3 \mathrm{Mpc}$. Our mid-IR:X-ray correlation from Eq. (2) then implies $\log L_{X}=42.39 \pm 0.06-$ the error being a $1-\sigma$ Monte Carlo uncertainty. In a recent determination of the intrinsic X-ray power based on the Fe $\mathrm{K} \alpha$ and the optical [OIII] $\lambda 5007$ emission lines, Levenson et al. (2006) estimate $\log L_{X}=42.40-42.81$ for ESO 428-G014 (after applying a small distance correction for consistency). This overlaps with the predicted $\log L_{X}$ from our correlation to within $1-\sigma$.

The mid-IR and X-ray observations for ESO 428-G014 are over-plotted in Fig. 1 and provide additional validation of our correlation in the $\mathrm{CT}$ regime. Of course, this result is based on a relatively-small sample of only eight CT sources, and further observations of an enlarged sample will be important for making these conclusions definitive.

There is one final noteworthy observation regarding star formation around CT AGN. If nuclear starbursts occur in disks aligned with the obscuring AGN tori, they should lie along our line-of-sight to the core and contaminate the nuclear flux, irrespective of angular-resolution; in this case a systematically higher $L_{\mathrm{IR}} / L_{\mathrm{X}}$ is expected in our $\mathrm{CT}$ point-like cores, which we do not observe. This seems to suggest the absence of any ongoing star formation (even out to several kpc) around local CT sources at the present epoch, at least to within the power limits that we discuss in Sect. 4.3.

\section{Summary}

In this work, we suggest that high-resolution mid-infrared continuum photometry is an accurate proxy for the intrinsic X-ray luminosity of local Seyferts. The emitted powers at $\sim 12-\mu \mathrm{m}$ and 2-10 keV are intimately correlated over three orders of magnitude in luminosity, and evidence suggests that this extends into the quasar regime. Interestingly, it has been noted in a preprint by Grossan (2004, based on high-resolution Keck imaging) that such a mid-IR:X-ray correlation may be valid for low-luminosity AGN as well. The suitability of the $10-12 \mu \mathrm{m}$ band for AGN selection has been emphasized by other earlier studies as well (e.g. Spinoglio \& Malkan 1989; Alonso-Herrero et al. 2001). Our tight correlation now extends this to the highest spatial resolution available, for Seyferts of all types including very heavily obscured sources, and places the conclusion on a precise, quantitative footing with very small scatter.

Combining our sample with Papers I and II, we have presented the most unbiased mid-IR fluxes to date of 41 AGN (not including NGC 1068, which was published by others) - 16 of these in the current paper. Selecting a subsample of sources based on a physically-motivated (but empirically-calibrated) threshold of physical scale resolution related to the torus size, we find the correlation to be especially tight: $L_{\mathrm{MIR}} \propto L_{\mathrm{X}}{ }^{1.11 \pm 0.07}$ (Eq. (2)) with additional systematic variation over the range of 1.02-1.21. We also find a well-defined range in mid-IR:X-ray spectral indices $\left(\alpha_{\mathrm{IX}}=-1.10 \pm 0.01\right)$ for this sample, and show that mid-IR bolometric corrections $\left(L_{\mathrm{Bol}} / L_{\mathrm{MIR}}\right.$, indirectly derived from $X$-rays) range over $\sim 10-30$ for the Sy luminosity range that we probe.

It is important to reiterate that even without any selection based on resolved scale, our full dataset still has a well-defined distribution in $L_{\mathrm{MIR}}$-vs.- $L_{\mathrm{X}}$ (Fig. 2). In fact, if one has access to mid-IR imaging of distant AGN (in effect, low-resolution imaging) and wishes to use this to determine intrinsic X-ray powers, then using the parameters for the correlation fit to all 42 sources (see "All" row in Table 2) is probably more appropriate. Similarly, if one wishes to predict total mid-IR fluxes for small-telescope imaging based on known $L_{X}$ values, the same parameters ought to be used. On the other hand, if the goal is to determine of the intrinsic mid-IR and X-ray emission of the AGN+torus, then the parameters for the well-resolved sample (i.e. Eq. (2)) can be used directly.

There are several other implications of our work and subsequent questions that we have touched upon only briefly in Papers II and herein. That mid-IR nuclear starbursts cannot be energetically-dominant within the central $\sim 70 \mathrm{pc}$ of local Seyfert nuclei was discussed in Sect. 4.3. Our tight dispersion implies that SBs account for no more than about $40 \%$ of the mid-IR emission that remains unresolved with the VLT, on average. This is to be considered as a strong upper limit, since other effects that increase the dispersion must also be present. The Herschel ${ }^{1}$ satellite (Poglitsch et al. 2006, scheduled for launch in 2009), with its large $3.5-\mathrm{m}$ diameter mirror and far-IR detectors, will provide an excellent opportunity to test this result with sensitive (spatial and spectral) deconvolution of SBs from AGN, better than any other space observatory to date. On a more immediate timescale, the Infrared Camera (IRC) on Japan's Akari $^{2}$ IR satellite has several filters sensitive around the $12-\mu \mathrm{m}$ regime, one of which (S9W) has been used for an all-sky survey more sensitive than IRAS by an order of magnitude (Murakami et al. 2007; Onaka et al. 2007; Ishihara et al. 2008). Our Seyfert correlation (based on a spatial resolution better than either of these satellites) will give a very accurate scaling for removal of contamination in broad-band spectral modeling of AGN with these and other future missions.

In this regard, we have not speculated on what the resolved scale threshold of $\sim 400-1700 r_{\text {sub }}$ (Sect. 5.1) could correspond to physically. If this is indeed $\sim 10$ times larger than typical outer torus radii found in recent models (Hönig et al. 2006), then it may instead correspond to a transition between inner and outer nuclear star-forming components (cf. Davies et al. 2007, who found a inner star formation scale of $\sim 50 \mathrm{pc}$ ). But more work is needed to verify this threshold and understand its origin.

Next, the increase in bolometric corrections - in other words, a decrease in $L_{\mathrm{MIR}} / L_{\mathrm{Bol}}$ - with increasing $L_{\mathrm{Bol}}$ is qualitatively

\footnotetext{
1 http://www.esa.int/science/herschel

2 http://www.ir.isas. jaxa.jp/ASTRO-F/
} 


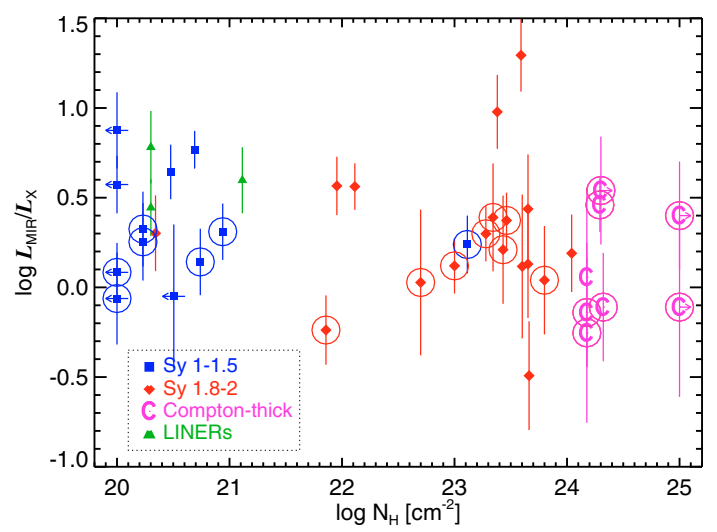

Fig. 8. Log luminosity-ratio as a function of obscuring column density determined from X-ray observations (for all sources excluding ESO 323-G032). $\left(\bar{r}, \sigma_{r}\right)=(0.24,0.38)$ and $(0.37,0.32)$ for the 24 obscured $\left(\log N_{\mathrm{H}} \geq 22\right)$ and 17 unobscured sources respectively. The corresponding values for the well-resolved sample are $(0.17,0.25)$ and $(0.12,0.21)$ with 15 and 7 sources respectively, with the two classes being indistinguishable according to a KS test. $N_{\mathrm{H}}$ uncertainties are not included in the plot, but this is irrelevant from the perspective of the absence of any trends. See Sect. 7. (This figure is available in color in electronic form.)

similar to what is inferred in the quasar regime by other authors (e.g. Maiolino et al. 2007; Treister et al. 2008) and may be interpreted in terms of a decrease in covering factor of dust for more powerful sources. Our correlation extends this result at least one order of magnitude into the Seyfert regime. Comparison with the steeper slope of the X-ray bolometric corrections with luminosity (Fig. 5) has consequences for the covering factor of obscuring gas. For instance, the nearly linear luminosity correlation may imply that both the gas and the dust are closely coupled at all powers. But we have not explored this in depth.

Finally, the fact that all types of Seyferts and Compton-thick AGN follow the same correlation suggests a similar origin for the mid-IR radiation in all these AGN classes. A nonthermal component is probably not dominant in most cases, as discussed in Sect. 4.2. It may be best explained in the context of the clumpy AGN tori discussed in Paper II (and references therein). This can also be emphasized by plotting the ratio of mid-IR:X-ray luminosities against the obscuring columns $N_{\mathrm{H}}$ : see Fig. 8 which is an update of Fig. 2 in Paper II. Any difference in the distribution of $\log \left(L_{\mathrm{MIR}} / L_{\mathrm{X}}\right)$ values for obscured sources with $\log N_{\mathrm{H}} \geq 22$, as compared to less obscured ones, is significant only at 78 per cent according to a KS test. Furthermore, the corresponding distributions for the well-resolved subsamples (obscured vs. unobscured) are completely indistinguishable. If the gas column density is a measure of the torus inclination angle to the lineof-sight, then smooth-dust torus models would predict a lower $\log \left(L_{\mathrm{MIR}} / L_{\mathrm{X}}\right)$ at high $N_{\mathrm{H}}$ values, which we do not see.

These issues must be accounted for when simulating radiative transfer through AGN tori. We leave detailed study to future work including modeling and observations of enlarged and more complete samples.

Acknowledgements. It is a pleasure to acknowledge the excellent support work by the staff at ESO that made the service-mode observations possible. We thank Eric Pantin for making his VISIR pipeline available to some of us. P.G. thanks Marco Ajello for discussion of his data of ESO 263-G013. He also acknowledges David R. Ballantyne, David M. Alexander, Richard F. Mushotzky, K. Tristram, and the referee for comments, criticisms and catching typos. P.G. was supported by JSPS and RIKEN Foreign Postdoctoral Researcher fellowships during the course of this work. A.C., R.G., and C.V. acknowledge partial support from the Italian Space Agency (contracts ASI-INAF I/023/05/0 and ASI I/088/06/0) and PRIN-MIUR (grant 2006-02-5203). This research has made use of: 1) the NASA/IPAC Extragalactic Database (NED), which is operated by the Jet Propulsion Laboratory, California Institute of Technology, under contract with the National Aeronautics and Space Administration; 2) the TARTARUS database, which is supported by Jane Turner and Kirpal Nandra under NASA grants NAG5-7385 and NAG5-7067; 3) the Multimission Archive at the Space Telescope Science Institute (MAST). STScI is operated by the Association of Universities for Research in Astronomy, Inc., under NASA contract NAS5-26555.

\section{Appendix A: Notes on individual sources}

\section{A.1. Compton-thin sources}

\section{A.1.1. SWIFT J0138.6-4001 and SWIFT J0601.9-8636}

The Sy type classification for SWIFT J0138.6-4001 comes from Bodaghee et al. (2007, and references therein); the one for SWIFT J0601.9-8636 from Landi et al. (2007). The two sources have only recently been identified as highly obscured AGN with very small scattering fractions by Ueda et al. (2007). Theirs is the best broad-band analysis (combining Suzaku and Swift data), and we use their luminosities directly after correction for transmission efficiency. Some variability (by factors of 1.6 and 0.5 respectively) as compared to the results of Tueller et al. (2008) was discussed by Ueda et al. (2007), so we assign a factor of 2 luminosity uncertainties.

SWIFT J0601.9-8636 was the least significant of all our VISIR targets, being barely detectable at the 5- $\sigma$ level. A single source, offset by $\Delta($ Right Ascension $)=-1$ ". $6, \Delta($ Declination $)=$ +2 .' 3 from the nominal position taken from the NASA Extragalactic Database, was found in several filters. Other VLT optical acquisition images obtained by us as part of a separate program confirm that this source coincides with the peak of the optical emission from this highly-reddened, edge-on galaxy.

Both targets were interpreted by Ueda et al. (2007) as being buried within geometrically-thick tori, and it is interesting to note that one of them (SWIFT J0138.6-4001) lies above the best-fit correlation line in Fig. 1 (i.e. is comparatively-weak at $12-\mu \mathrm{m}$, for its X-ray luminosity), possibly implying that hot dust clouds are smothered within the thick tori and not observed directly. Sensitive observations over a broad wavelength range will be required for confirming the nature of this source.

\section{A.1.2. ESO 209-G012}

Sazonov et al. (2007) state $F_{17-60 \mathrm{keV}}=1.66 \times$ $10^{-11} \mathrm{erg} \mathrm{s}^{-1} \mathrm{~cm}^{-2}=>\log L_{17-60}=43.75$ and $\log N_{\mathrm{H}}<22$ based on their INTEGRAL and ROSAT analysis. Using XSPEC (Arnaud 1996) and assuming an unabsorbed power law (PL) model with $\Gamma=1.7$ and the above normalization also agrees well with $L_{20-100}$ stated by Bassani et al. (2006) when extrapolated to higher energies. This PL then implies $L_{2-10}=4.45 \times 10^{43} \mathrm{erg} \mathrm{s}^{-1}$. The model straddles the $20-40 \mathrm{keV}$ luminosities stated by Bird et al. (2006) and Bird et al. (2007), thus giving a simple uncertainty estimate between these.

\section{A.1.3. NGC 3081}

From a combined Swift and INTEGRAL analysis, Beckmann et al. (2007) find $\Gamma=1.8 \pm 0.2$ with $L_{20-100}=8.1 \times 10^{42} \mathrm{erg} \mathrm{s}^{-1}$. They also find significant variability of a factor of a few in the BAT 14-195 keV lightcurve over a 15 month period.

Fitting an absorbed PL above a few keV (the softer flux is fit by weak scattering or a blackbody) to spectra extracted from the Tartarus database gives $N_{\mathrm{H}}=68( \pm 5) \times 10^{22} \mathrm{~cm}^{-2}$ with 
$L_{2-10}=4-6 \times 10^{42} \mathrm{erg} \mathrm{s}^{-1}$, the variation being the one between the ASCA SISs. Extending the de-absorbed PL to $200 \mathrm{keV}$ gives $L_{14-195}=9-14 \times 10^{42} \mathrm{erg} \mathrm{s}^{-1}$, matching the more recent values quoted by Markwardt et al. (2005) and Ajello et al. (2008). This suggests that variability may not be very strong.

On the other hand, BeppoSAX is known to have observed the source in a low flux state (Maiolino et al. 1998). We take the lower value of the luminosity range found in the above ASCA fits, with a large error of $\log L=0.3$ as a mean value.

This source was also imaged by Krabbe et al. (2001) at 1 ". 3 resolution. The full $N$-band flux quoted by them is consistent with our narrow-band PAH2 flux (assuming a flat SED in $\lambda F_{\lambda}$ units), implying the absence of extended emission on $\sim 1^{\prime \prime}$ scales.

\section{A.1.4. ESO 263-G013}

The only good-quality X-ray spectrum published is the Swift XRT analysis by Landi et al. (2007), who find $N_{\mathrm{H}}=4 \pm 1 \times$ $10^{23} \mathrm{~cm}^{-2}$ absorbing a PL with $\Gamma \approx 2.32$. The observed flux is $F_{2-10}=2 \times 10^{-12} \mathrm{erg} \mathrm{s}^{-1} \mathrm{~cm}^{-2}$ and they quote an intrinsic $L_{2-10}=4-7 \times 10^{42} \mathrm{erg} \mathrm{s}^{-1}$ (converted to our cosmology). But extending this steep PL to higher energies underpredicts other INTEGRAL and Swift fluxes by factors of $\sim 7-10$ (Bird et al. 2007; Ajello et al. 2006). Recent XMM-Newton data (by M. Ajello, priv. comm., and some of us) analyzed in conjunction with the Swift BAT data are instead fit with a harder photonindex $\Gamma \approx 1.8$, resulting in reasonable agreement (to within a factor of $\sim 2$ ) with fluxes over $2-100 \mathrm{keV}$ and an intrinsic $L_{2-10} \sim 2 \times 10^{43} \mathrm{erg} \mathrm{s}^{-1}$; we prefer this latter broad-band analysis.

\section{A.1.5. NGC 4388}

Combined analysis from several missions shows a simple PL above a few keV absorbed by a column $N_{\mathrm{H}}=2.7 \times 10^{23} \mathrm{~cm}^{-2}$, but with highly variable fluxes and obscuring column (Beckmann et al. 2004; Cappi et al. 2006; Iwasawa et al. 2003; Risaliti et al. 2002). Using the mean of the broad range found in $X M M-N e w t o n$ by Cappi et al. (2006) and recent INTEGRAL results by Beckmann et al. (2006), for a distance of $16.7 \mathrm{Mpc}$, results in a mean $\log L_{2-10}=42.24$, to which we attach a large $1-\sigma$ error in dex of 0.3 .

\section{A.1.6. ESO 323-G032}

This source is classified as a Sy 1.9 in the catalog of Véron-Cetty \& Véron (2001). The only reliable published X-ray measurements happen to be the INTEGRAL observations of Bird et al. (2007), who find $F_{20-40}=6.81 \times 10^{-12}$ and $F_{40-100}=1.51 \times$ $10^{-11} \mathrm{erg} \mathrm{s}^{-1} \mathrm{~cm}^{-2}$. In the absence of other information, we parametrize this with a simple PL, $\Gamma \approx 1.2$, which extrapolates to $F_{2-10}=3.7 \times 10^{-12} \mathrm{erg} \mathrm{s}^{-1} \mathrm{~cm}^{-2}$, or $L_{2-10}=2.0 \times$ $10^{42} \mathrm{erg} \mathrm{s}^{-1}$. One could also predict the $2-10 \mathrm{keV}$ power based on forbidden optical emission line measurements. Correcting the [OIII] $\lambda 5007$ emission line flux from Gu et al. (2006) for a small extinction factor from the same paper, and using the type-1 $\left\langle F_{2-10} / F_{[\mathrm{OIII}]}\right\rangle$ relation from Panessa et al. (2006), we get $L_{2-10} \approx 3.3 \times 10^{42} \mathrm{erg} \mathrm{s}^{-1}$.

\section{A.1.7. NGC 4992}

The Sy classification of this source comes from Bodaghee et al. (2007) and references therein. Comastri et al. (2007) present a broad-band Suzaku spectral analysis, and infer an extreme reflection component with $R>5$ and a possible relativistically-blurred Fe line, indicative of disk-dominated reflection. This can be naturally explained by relativistic light-bending on to the inner disk, which enhances reflection and suppresses the incident power law at infinity (Miniutti et al. 2004). The fraction of AGN described by this light-bending model is still unknown, though increasing numbers of sources are being discovered, and the hard X-ray background spectrum can also accommodate a non-negligible fraction of light-bent source in the Universe (see for instance Miniutti et al. 2004; Gandhi et al. 2007; Miniutti et al. 2007, and references therein).

Although the majority of intrinsic power law photons may be bent towards the event horizon and never escape the black hole's gravitational sphere of influence, the relevant luminosity in our case is the X-ray flux that is incident on the distant torus clouds, i.e. the (absorption-corrected) power observed at infinity. Using the model of Comastri et al. (2007) yields $L_{2-10}=1.7 \times 10^{43} \mathrm{erg} \mathrm{s}^{-1}$. If part of the high reflection stems instead from decreased transmission efficiency by heavy obscuring material or from strong time variability, then this estimate will be a lower limit (cf. Ueda et al. 2007).

\section{A.1.8. NGC 6300}

This is one of a sample of "changing-look" AGN whose spectra exhibit transitions between Compton-thick and Compton-thin obscuration on timescales of years (Matt et al. 2003). It was found to be reflection-dominated in 1997, but has since been in a Compton-thin state (RXTE and BeppoSAX analysis; Guainazzi 2002). Assuming a Compton-thin column of $N_{\mathrm{H}}=2 \times 10^{23} \mathrm{~cm}^{-2}$ (from the XMM-Newton analysis of Matsumoto et al. 2004) obscuring a PL with $\Gamma=2.42$ (Beckmann et al. 2006) and normalizing to JEM-X and ISGRI $2-100 \mathrm{keV}$ fluxes of the later publication, the implied intrinsic $\log L_{2-10}=42.76$. But, in addition to its changing-look nature, the source also exhibits large flux variability (Awaki et al. 2006), and a much lower luminosity of $\log L_{2-10}=41.8$ was derived by Matsumoto et al. (2004) from their XMM-Newton analysis. We use a mean $\log L_{2-10}=42.3$.

\section{A.1.9. ESO 103-G035}

Molina et al. (2006) analyzed the broad-band INTEGRAL spectrum of this source and located an exponential cut-off to the X-ray PL at $E_{\text {cut }} \approx 70 \mathrm{keV}$, with no substantial flux change from previous BeppoSAX observations (Risaliti 2002; Wilkes et al. 2001). This source has shown moderate and fast $N_{\mathrm{H}}$ variability on timescales of several months (Risaliti et al. 2002). Using model realizations in XSPEC, we find that the PL model of Molina et al. (2006) with $\Gamma=1.78, N_{\mathrm{H}}=2 \times 10^{23} \mathrm{~cm}^{-1}$ and $F_{2-10}=3 \times 10^{-11} \mathrm{~cm}^{-2}$ is consistent with the higher energy 20-100 keV flux when extrapolated, but slightly underestimates (by $\sim 30$ per cent) the latest $20-40$ and $40-100 \mathrm{keV}$ fluxes from Bird et al. (2007). On the other hand, the Swift BAT 14-195 keV luminosity is overestimated by a factor of $\approx 2$. We assume the base $2-10 \mathrm{keV}$ luminosity of the Molina et al. model, with an $1-\sigma$ luminosity error of 0.15 in dex.

\section{A.1.10. NGC 6814}

This source is an unabsorbed Sy 1.5 , with $\log N_{\mathrm{H}}<20.7$ (Reynolds 1997). It is known to be highly variable, even after accounting for a neighboring $\mathrm{CV}$ that contaminated early 
observations (e.g. see the results of long-term RXTE monitoring described by Mukai et al. 2003). Using the PL model with $\Gamma=2.48$ from Beckmann et al. (2006) and extrapolating from their INTEGRAL 20-100 fluxes to below $10 \mathrm{keV}$ suggests that JEM-X should have detected the source, which it did not. Instead, some spectral curvature probably needs to be invoked. Including a simple PEXRAV (Magdziarz \& Zdziarski $1995)$ parametrization with $\Gamma=1.6, R=1$ and $E_{\text {cut }}=150 \mathrm{keV}$ agrees with $20-40 \mathrm{keV}$ and $40-100 \mathrm{keV}$ fluxes of Bird et al. (2007) and with the mean published RXTE observations mentioned above, and also matches the $<10 \mathrm{keV}$ photon-index found from ASCA analysis of data in the Tartarus database. This implies a mean $L_{2-10}=1.33 \times 10^{42} \mathrm{erg} \mathrm{s}^{-1}$. A large variation of 0.4 in dex accounts for the source variability.

\section{A.2. Compton-thick sources}

\section{A.2.1. NGC 1068}

NGC 1068 is one of the best-studied of obscured AGN; hence our decision to include it on our correlation. In X-rays, it is known to be substantially Compton-thick and reflectiondominated, with $\log N_{\mathrm{H}}>25$ (Matt et al. 2000; Iwasawa et al. 1997; Koyama et al. 1989). By correcting from the observed flux of X-rays scattered from above the obscuring medium into the line-of-sight or from the flux of the forbidden [OIII] $\lambda 5007 \AA$ emission line, intrinsic $\log L_{2-10}$ estimates of $\sim 42.8-44$ are derived (Iwasawa et al. 2003; Panessa et al. 2006; Levenson et al. 2006).

In the mid-IR, several high-resolution studies have been carried out from the ground revealing bright, extended structures on sub-arcsec scales and beyond (Galliano et al. 2005b; Bock et al. 2000; Alloin et al. 2000). At the highest spatial sampling, continuum core fluxes of 9-10 Jy have been reported around $12-\mu \mathrm{m}$ (Mason et al. 2006; Tomono et al. 2001), and they correspond to $\log \lambda L_{\lambda} \approx 43.8$.

\section{A.2.2. NGC 3281}

Vignali \& Comastri (2002) identify this source as mildly Compton-thick. Using their model (c), which corrects for Compton-scattering in a physically-motivated way implies $L_{2-10}=1.7 \times 10^{43}$ and $L_{20-100}=2.1 \times 10^{43} \mathrm{erg} \mathrm{s}^{-1}$ in our cosmology. Using this model to infer the $20-40 \mathrm{keV}$ and 14-195 keV fluxes in order to compare with the recent studies of Bird et al. (2007) and Markwardt et al. (2005) suggests $\log L_{2-10}=43.30 \pm 0.15$.

Krabbe et al. (2001) detected extended $N$-band emission beyond their resolution limit of $1^{\prime \prime}$ in this source. Consistent with this, our 0 .'35-resolution flux (in units of $\lambda F_{\lambda}$ ) is even lower than theirs by $\approx 30$ per cent.

\section{A.2.3. NGC 3393}

Corrections based on the $\mathrm{Fe}$ line and on the flux of forbidden optical [OIII] emission yield $L_{2-10} \approx 7.2-17 \times 10^{42} \mathrm{erg} \mathrm{s}^{-1}$ (Levenson et al. 2006). Combining XMM-Newton analysis with previous BeppoSAX data, Guainazzi et al. (2005) find a decreased column density $\left(N_{\mathrm{H}} \sim 4 \times 10^{24} \mathrm{~cm}^{-2}\right)$ with respect to the complete Compton-thick suppression $\left(N_{\mathrm{H}}>10^{25} \mathrm{~cm}^{-2}\right)$ inferred by Maiolino et al. (1998). Because only a small part of the broad-band X-ray flux is transmitted, correcting the observed continuum to estimate the intrinsic luminosity is highly uncertain given the current data quality on this source. Still,
Levenson et al. (2006) find reasonable agreement when extrapolating the BeppoSAX high-energy data to their Chandra bandpasses with $\Gamma \approx 2$. Suzaku may provide better estimates in the near future.

\section{A.2.4. IC 3639}

The intrinsic high-energy flux from this source is likely to be completely depleted by heavy Compton-thick obscuration with $N_{\mathrm{H}}>10^{25} \mathrm{~cm}^{-2}$ (e.g., Risaliti et al. 1999). Analysis is complicated by most of the $<10 \mathrm{keV}$ observed X-ray flux probably being related to extended emission (Ghosh et al. 2007; Guainazzi et al. 2005b). Using instead the [OIII] $\lambda 5007$ emission line $\left(F_{\text {[OIII] }}=3.4 \times 10^{-12} \mathrm{erg} \mathrm{s}^{-1} \mathrm{~cm}^{-2}\right.$ from Lumsden et al. 2001, corrected for extinction) as a proxy for the intrinsic power (in conjunction with the [OIII]:X-ray relation from Panessa et al. 2006) gives $L_{2-10} \sim 4 \times 10^{43} \mathrm{erg} \mathrm{s}^{-1}$. This also matches older [OIII] measurements quoted by Guainazzi et al. (2005b), if we correct for extinction using the prescription of Bassani et al. (1999).

\section{A.2.5. NGC 5728}

From a broad-band Suzaku analysis, Comastri et al. (2007) conclude that this source is Compton-thick with $N_{\mathrm{H}}=$ $2.1 \times 10^{24} \mathrm{~cm}^{-2}$. The unabsorbed flux is $F_{2-10} \sim 5 \times$ $10^{-11} \mathrm{erg} \mathrm{s}^{-1} \mathrm{~cm}^{-2}$, implying $L_{2-10} \sim 8.7 \times 10^{42} \mathrm{erg} \mathrm{s}^{-1}$. There is additional low-energy spectral complexity due to scattering/reflection, but this accounts for only 1-2 per cent of the 2-10 keV power. The mildly Compton-thick column suppresses the overall X-ray continuum by only $\sim 5-10$ per cent; we include these small corrections.

From the raw [OIII] $\lambda 5007$ optical emission line measurement of $\mathrm{Gu}$ et al. (2006) along with the type- $1\left\langle F_{2-10} / F_{[\mathrm{OIII}]}\right\rangle$ relation from Panessa et al. (2006), we derive $L_{2-10} \sim 1.1 \times$ $10^{42} \mathrm{erg} \mathrm{s}^{-1}$. Correcting for extinction using the Balmer decrement yields $L_{2-10} \sim 3.4 \times 10^{42} \mathrm{erg} \mathrm{s}^{-1}$.

We use the mean of the above luminosities, which also agrees with the extrapolated Swift BAT measurement by Markwardt et al. (2005), within errors.

\section{A.2.6. ESO 138-G001}

Collinge \& Brandt (2000) found evidence for Compton-thick obscuration in this source, based on their ASCA spectral analysis and comparison with [OIII] optical emission line flux, but the reflection fraction and obscuring column were not wellconstrained. Suppose we assume a model with a PL $(\Gamma=2.1)$ obscured by a mildly Compton-thick $N_{\mathrm{H}}=2 \times 10^{24} \mathrm{~cm}^{-2}$, a reflection component $R=1$, and a typical high energy exponential cut-off at $200 \mathrm{keV}$. Then, normalizing the model to recent INTEGRAL 20-40 keV fluxes from Bird et al. (2007) leads to a good match to reported measurements up to $100 \mathrm{keV}$ (Bassani et al. 2006). In this case, $\log L_{2-10}=42.9$ intrinsically, including mild correction for multiple Compton down-scattering (Wilman \& Fabian 1999).

Using instead the Sy 1 relationship between the [OIII] and 2-10 keV fluxes described by Collinge \& Brandt (2000) results in a luminosity that is only slightly higher, at $\log L_{2-10}=43.10$. Given the paucity of good X-ray data on this source, we assume the mean of the above luminosities, with a large uncertainty of 0.3 dex. 
Appendix B: Full data list

Table B.1. Full sample from Papers I, II and present paper, used in the correlation fits.

\begin{tabular}{|c|c|c|c|c|c|c|c|c|}
\hline Source & Type & $\begin{array}{c}\text { Distance } \\
\text { Mpc } \\
\text { (3) }\end{array}$ & $\begin{array}{l}r_{0} \\
\mathrm{pc} \\
(4)\end{array}$ & Filter & $\begin{array}{l}\text { Flux } \\
\text { mJy } \\
(6)\end{array}$ & $\begin{array}{c}\log L_{\mathrm{MIR}} \\
\mathrm{erg} \mathrm{s}^{-1} \\
(7)\end{array}$ & $\begin{array}{c}\log L_{\mathrm{X}} \\
\mathrm{erg} \mathrm{s}^{-1} \\
(8)\end{array}$ & $\alpha_{\mathrm{IX}}$ \\
\hline Fairall 9 & 1.2 & 206 & 320 & NEII & $306 \pm 10$ & $44.51 \pm 0.03$ & $43.87 \pm 0.15$ & -1.21 \\
\hline NGC 526A & 1.9 & 81 & 135 & NEII_1 & $275 \pm 55$ & $43.70 \pm 0.08$ & $43.14 \pm 0.10$ & -1.21 \\
\hline SWIFT J0138.6-4001 & 2 & 108 & 175 & PAH2 & $34 \pm 3$ & $43.10 \pm 0.04$ & $43.59 \pm 0.30$ & -0.96 \\
\hline Mrk 590 & 1 & 112 & 175 & NEII & $106 \pm 13$ & $43.55 \pm 0.06$ & $43.61 \pm 0.25$ & -1.06 \\
\hline NGC 1068 & CT & 14 & 25 & * & $9500 \pm 475$ & $43.80 \pm 0.03$ & $43.40 \pm 0.30$ & -1.16 \\
\hline NGC 1097 & $\mathrm{~L}$ & 17 & 30 & NEII_1 & $28 \pm 7$ & $41.40 \pm 0.11$ & $40.80 \pm 0.15$ & -1.22 \\
\hline SWIFT J0601.9-8636 & 2 & 25 & 45 & NEII_2 & $72 \pm 15$ & $42.11 \pm 0.08$ & $41.92 \pm 0.30$ & -1.10 \\
\hline ESO 209-G012 & 1.5 & 179 & 280 & NEII & $188 \pm 26$ & $44.22 \pm 0.06$ & $43.65 \pm 0.15$ & -1.21 \\
\hline NGC 3081 & $1 \mathrm{~h}$ & 34 & 55 & NEII_2 & $138 \pm 11$ & $42.64 \pm 0.04$ & $42.60 \pm 0.30$ & -1.07 \\
\hline ESO 263-G013 & 2 & 143 & 225 & NEII & $46 \pm 3$ & $43.42 \pm 0.02$ & $43.30 \pm 0.40$ & -1.09 \\
\hline NGC 3281 & CT & 46 & 75 & NEII_2 & $1016 \pm 52$ & $43.76 \pm 0.02$ & $43.30 \pm 0.15$ & -1.16 \\
\hline NGC 3393 & CT & 53 & 90 & NEII_2 & $94 \pm 5$ & $42.90 \pm 0.02$ & $43.04 \pm 0.30$ & -1.01 \\
\hline NGC 3783 & 1.5 & 43 & 70 & NEII_1 & $722 \pm 67$ & $43.52 \pm 0.05$ & $43.21 \pm 0.15$ & -1.15 \\
\hline NGC 4388 & $1 \mathrm{~h}$ & 17 & 30 & NEII_2 & $375 \pm 28$ & $42.45 \pm 0.03$ & $42.24 \pm 0.30$ & -1.11 \\
\hline NGC 4507 & $1 \mathrm{~h}$ & 51 & 80 & NEII_1 & $685 \pm 50$ & $43.67 \pm 0.04$ & $43.30 \pm 0.15$ & -1.17 \\
\hline NGC 4579 & $\mathrm{~L}$ & 21 & 35 & NEII_1 & $61 \pm 21$ & $41.88 \pm 0.14$ & $41.10 \pm 0.15$ & -1.26 \\
\hline NGC 4593 & 1 & 38 & 65 & NEII_1 & $382 \pm 73$ & $43.18 \pm 0.08$ & $42.93 \pm 0.20$ & -1.13 \\
\hline IC 3639 & CT & 46 & 75 & NEII_2 & $542 \pm 29$ & $43.51 \pm 0.02$ & $43.62 \pm 0.50$ & -1.04 \\
\hline ESO 323-G032 & 1.9 & 68 & 110 & PAH2 & $61 \pm 6$ & $42.96 \pm 0.04$ & $42.43 \pm 0.40$ & -1.24 \\
\hline NGC 4941 & 2 & 17 & 30 & NEII_1 & $81 \pm 6$ & $41.74 \pm 0.05$ & $41.30 \pm 0.30$ & -1.18 \\
\hline NGC 4992 & 2 & 108 & 175 & NEII & $71 \pm 4$ & $43.36 \pm 0.02$ & $43.23 \pm 0.30$ & -1.09 \\
\hline IRAS 13197-1627 & $1 \mathrm{~h}$ & 73 & 115 & NEII_1 & $875 \pm 46$ & $44.07 \pm 0.03$ & $42.78 \pm 0.20$ & -1.31 \\
\hline Cen A & 2 & 3.8 & 7 & NEII_1 & $1451 \pm 73$ & $41.80 \pm 0.04$ & $41.68 \pm 0.15$ & -1.10 \\
\hline NGC 5135 & CT & 60 & 95 & NEII_1 & $123 \pm 12$ & $43.06 \pm 0.05$ & $43.00 \pm 0.50$ & -1.07 \\
\hline MCG-06-30-15 & 1.5 & 34 & 55 & NEII_1 & $393 \pm 49$ & $43.07 \pm 0.06$ & $42.74 \pm 0.20$ & -1.10 \\
\hline NGC 5728 & CT & 38 & 65 & NEII_2 & $123 \pm 10$ & $42.69 \pm 0.03$ & $42.80 \pm 0.30$ & -1.03 \\
\hline NGC 5995 & 2 & 108 & 170 & NEII & $421 \pm 61$ & $44.11 \pm 0.06$ & $43.54 \pm 0.15$ & -1.20 \\
\hline ESO 138-G001 & $\mathrm{CT}$ & 38 & 65 & NEII_2 & $862 \pm 45$ & $43.54 \pm 0.02$ & $43.00 \pm 0.30$ & -1.17 \\
\hline NGC 6300 & 2 & 16 & 25 & NEII_2 & $727 \pm 38$ & $42.69 \pm 0.02$ & $42.30 \pm 0.30$ & -1.11 \\
\hline ESO 103-G035 & 2 & 57 & 95 & NEII_2 & $622 \pm 49$ & $43.74 \pm 0.03$ & $43.44 \pm 0.15$ & -1.14 \\
\hline ESO 141-G055 & 1 & 156 & 240 & NEII_1 & $170 \pm 47$ & $44.04 \pm 0.11$ & $43.90 \pm 0.15$ & -1.10 \\
\hline NGC 6814 & 1.5 & 21 & 35 & NEII_2 & $96 \pm 6$ & $42.07 \pm 0.02$ & $42.12 \pm 0.40$ & -1.07 \\
\hline Mrk 509 & 1.5 & 147 & 225 & NEII & $269 \pm 42$ & $44.18 \pm 0.07$ & $44.10 \pm 0.15$ & -1.11 \\
\hline PKS 2048-57 & $1 \mathrm{~h}$ & 47 & 80 & NEII_1 & $1035 \pm 108$ & $43.82 \pm 0.05$ & $42.84 \pm 0.20$ & -1.30 \\
\hline PG 2130+099 & 1.5 & 274 & 415 & NEII & $179 \pm 31$ & $44.53 \pm 0.07$ & $43.65 \pm 0.20$ & -1.24 \\
\hline NGC 7172 & 2 & 38 & 60 & NEII_1 & $165 \pm 27$ & $42.79 \pm 0.07$ & $42.76 \pm 0.40$ & -1.09 \\
\hline NGC 7213 & $\mathrm{~L}$ & 25 & 40 & NEII_1 & $271 \pm 27$ & $42.67 \pm 0.05$ & $42.23 \pm 0.15$ & -1.18 \\
\hline $3 \mathrm{C} 445$ & 1.5 & 247 & 365 & NEII & $206 \pm 28$ & $44.50 \pm 0.06$ & $44.26 \pm 0.15$ & -1.16 \\
\hline NGC 7314 & $1 \mathrm{~h}$ & 21 & 35 & PAH2 & $75 \pm 22$ & $41.96 \pm 0.12$ & $42.20 \pm 0.15$ & -1.01 \\
\hline NGC 7469 & 1.5 & 68 & 110 & NEII_1 & $627 \pm 35$ & $43.92 \pm 0.03$ & $43.15 \pm 0.10$ & -1.24 \\
\hline NGC 7674 & CT & 125 & 195 & NEII & $506 \pm 29$ & $44.31 \pm 0.03$ & $44.56 \pm 0.50$ & -1.00 \\
\hline NGC 7679 & 2 & 73 & 115 & NEII_1 & $46 \pm 18$ & $42.82 \pm 0.15$ & $42.52 \pm 0.15$ & -1.14 \\
\hline
\end{tabular}

Targets listed in order of increasing right ascension. In Col. (2), L = LINER and CT = Compton-thick. Column (4) lists approximate physical scale corresponding to an angular resolution limit of $0{ }^{\prime} 35$. * For NGC 1068, observations around $12 \mu \mathrm{m}$ from Tomono et al. (2001) and Mason et al. (2006) were used. Refer to Table 1 and the text for other details.

\section{References}

Ajello, M., Greiner, J., Küpcü Yoldas, A., et al. 2006, The Astronomer's Telegram, 864, 1

Ajello, M., Rau, A., Greiner, J., et al. 2008, ApJ, 673, 96

Alexander, D. M., Chary, R.-R., Pope, A., et al. 2008, ApJ, 687, 835

Alloin, D., Pantin, E., Lagage, P. O., \& Granato, G. L. 2000, A\&A, 363, 926

Alonso-Herrero, A., Quillen, A. C., Simpson, C., Efstathiou, A., \& Ward, M. J. 2001, AJ, 121, 1369

Arnaud, K. A. 1996, in Astronomical Data Analysis Software and Systems V, ed. G. H. Jacoby, \& J. Barnes, ASP Conf. Ser., 101, 5, 17

Awaki, H., Murakami, H., Ogawa, Y., \& Leighly, K. M. 2006, ApJ, 645, 928

Ballantyne, D. R. 2008, ApJ, 685, 787

Ballantyne, D. R., \& Papovich, C. 2007, ApJ, 660, 988

Barvainis, R. 1987, ApJ, 320, 537

Bassani, L., Dadina, M., Maiolino, R., et al. 1999, ApJS, 121, 473
Bassani, L., Molina, M., Malizia, A., et al. 2006, ApJ, 636, L65 Beckmann, V., Gehrels, N., Favre, P., et al. 2004, ApJ, 614, 641 Beckmann, V., Gehrels, N., Shrader, C. R., \& Soldi, S. 2006, ApJ, 638, 642 Beckmann, V., Gehrels, N., \& Tueller, J. 2007, ApJ, 666, 122

Bianchi, S., Chiaberge, M., Piconcelli, E., Guainazzi, M., \& Matt, G. 2008, MNRAS, 386, 105

Bird, A. J., Barlow, E. J., Bassani, L., et al. 2006, ApJ, 636, 765

Bird, A. J., Malizia, A., Bazzano, A., et al. 2007, ApJS, 170, 175

Bock, J. J., Neugebauer, G., Matthews, K., et al. 2000, AJ, 120, 2904

Bodaghee, A., Courvoisier, T. J.-L., Rodriguez, J., et al. 2007, A\&A, 467, 585

Bohlin, R. C., Savage, B. D., \& Drake, J. F. 1978, ApJ, 224, 132

Brandt, W. N., Fabian, A. C., Takahashi, K., et al. 1997, MNRAS, 290, 617

Buchanan, C. L., Gallimore, J. F., O’Dea, C. P., et al. 2006, AJ, 132, 401

Cappi, M., Panessa, F., Bassani, L., et al. 2006, A\&A, 446, 459

Carleton, N. P., Elvis, M., Fabbiano, G., et al. 1987, ApJ, 318, 595 
Cid Fernandes, R., Gu, Q., Melnick, J., et al. 2004, MNRAS, 355, 273 Collinge, M. J., \& Brandt, W. N. 2000, MNRAS, 317, L35

Comastri, A., Gilli, R., Vignali, C., et al. 2007, Progress of Theoretical Physics Supplement, 169, 274

Davies, R. I., Mueller Sánchez, F., Genzel, R., et al. 2007, ApJ, 671, 1388

Draine, B. T. 2003, ARA\&A, 41, 241

Dullemond, C. P., \& van Bemmel, I. M. 2005, A\&A, 436, 47

Elvis, M., Wilkes, B. J., McDowell, J. C., et al. 1994, ApJS, 95, 1

Fabian, A. C., \& Iwasawa, K. 1999, MNRAS, 303, L34

Fiore, F., Puccetti, S., Brusa, M., et al. 2009, ApJ, 693, 447

Galliano, E., Alloin, D., Pantin, E., Lagage, P. O., \& Marco, O. 2005a, A\&A, 438,803

Galliano, E., Pantin, E., Alloin, D., \& Lagage, P. O. 2005b, MNRAS, 363, L1

Gandhi, P., \& Fabian, A. C. 2003, MNRAS, 339, 1095

Gandhi, P., Fabian, A. C., Suebsuwong, T., et al. 2007, MNRAS, 382, 1005

Ghosh, H., Pogge, R. W., Mathur, S., Martini, P., \& Shields, J. C. 2007, ApJ, 656,105

Gilli, R., Comastri, A., \& Hasinger, G. 2007, A\&A, 463, 79

González Delgado, R. M., Heckman, T., Leitherer, C., et al. 1998, ApJ, 505, 174

González Delgado, R. M., Heckman, T., \& Leitherer, C. 2001, ApJ, 546, 845

Granato, G. L., Danese, L., \& Franceschini, A. 1997, ApJ, 486, 147

Grossan, B. 2004 [arXiv:astro-ph/0405190]

Gu, Q., Melnick, J., Fernandes, R. C., et al. 2006, MNRAS, 366, 480

Guainazzi, M. 2002, MNRAS, 329, L13

Guainazzi, M., Fabian, A. C., Iwasawa, K., Matt, G., \& Fiore, F. 2005, MNRAS, 356,295

Guainazzi, M., Matt, G., \& Perola, G. C. 2005b, A\&A, 444, 119

Hao, L., Weedman, D. W., Spoon, H. W. W., et al. 2007, ApJ, 655, L77

Hönig, S. F., Beckert, T., Ohnaka, K., \& Weigelt, G. 2006, A\&A, 452, 459

Hönig, S. F., Prieto, M. A., \& Beckert, T. 2008a, A\&A, 485, 33

Hönig, S. F., Smette, A., Beckert, T., et al. 2008b, A\&A, 485, L21

Hopkins, P. F., Richards, G. T., \& Hernquist, L. 2007, ApJ, 654, 731

Horst, H., Smette, A., Gandhi, P., \& Duschl, W. J. 2006, A\&A, 457, L17 (Paper I)

Horst, H., Gandhi, P., Smette, A., \& Duschl, W. J. 2008, A\&A, 479, 389 (Paper II)

Horst, H., Duschl, W. J., Gandhi, P., \& Smette, A. 2009, A\&A, 495, 137 (Paper III)

Imanishi, M. 2002, ApJ, 569, 44

Ishihara, D., Kataza, H., Yamamura, I., et al. 2008, in COSPAR, Plenary Meeting, 37th COSPAR Scientific Assembly, 37, 1325

Isobe, T., Feigelson, E. D., Akritas, M. G., \& Babu, G. J. 1990, ApJ, 364, 104 Iwasawa, K., Fabian, A. C., \& Matt, G. 1997, MNRAS, 289, 443

Iwasawa, K., Wilson, A. S., Fabian, A. C., \& Young, A. J. 2003, MNRAS, 345, 369

Jaffe, W., Meisenheimer, K., Röttgering, H. J. A., et al. 2004, Nature, 429, 47

Kishimoto, M., Hönig, S. F., Beckert, T., \& Weigelt, G. 2007, A\&A, 476, 713

Koyama, K., Inoue, H., Tanaka, Y., et al. 1989, PASJ, 41, 731

Krabbe, A., Böker, T., \& Maiolino, R. 2001, ApJ, 557, 626

Lagage, P. O., Pel, J. W., Authier, M., et al. 2004, The Messenger, 117, 12

Landi, R., Masetti, N., Morelli, L., et al. 2007, ApJ, 669, 109

Levenson, N. A., Heckman, T. M., Krolik, J. H., Weaver, K. A., \& Życki, P. T. 2006, ApJ, 648, 111

Lumsden, S. L., Heisler, C. A., Bailey, J. A., Hough, J. H., \& Young, S. 2001, MNRAS, 327, 459

Lutz, D., Maiolino, R., Spoon, H. W. W., \& Moorwood, A. F. M. 2004, A\&A, 418, 465

Magdziarz, P., \& Zdziarski, A. A. 1995, MNRAS, 273, 837

Maiolino, R., Ruiz, M., Rieke, G. H., \& Keller, L. D. 1995, ApJ, 446, 561

Maiolino, R., Salvati, M., Bassani, L., et al. 1998, A\&A, 338, 781

Maiolino, R., Marconi, A., Salvati, M., et al. 2001, A\&A, 365, 28

Maiolino, R., Shemmer, O., Imanishi, M., et al. 2007, A\&A, 468, 979

Marconi, A., Risaliti, G., Gilli, R., et al. 2004, MNRAS, 351, 169

Markwardt, C. B., Tueller, J., Skinner, G. K., et al. 2005, ApJ, 633, L77

Mason, R. E., Geballe, T. R., Packham, C., et al. 2006, ApJ, 640, 612

Mateos, S., Barcons, X., Carrera, F. J., et al. 2005, A\&A, 444, 79

Matsumoto, C., Nava, A., Maddox, L. A., et al. 2004, ApJ, 617, 930

Matt, G., Fabian, A. C., Guainazzi, M., et al. 2000, MNRAS, 318, 173

Matt, G., Guainazzi, M., \& Maiolino, R. 2003, MNRAS, 342, 422
McKernan, B., Ford, K. E. S., Chang, N., \& Reynolds, C. S. 2009, MNRAS, 394,491

Meisenheimer, K., Tristram, K. R. W., Jaffe, W., et al. 2007, A\&A, 471, 453

Meléndez, M., Kraemer, S. B., Armentrout, B. K., et al. 2008, ApJ, 682, 94

Miniutti, G., Fabian, A. C., \& Miller, J. M. 2004, MNRAS, 351, 466

Miniutti, G., Ponti, G., Dadina, M., Cappi, M., \& Malaguti, G. 2007, MNRAS, 375,227

Molina, M., Malizia, A., Bassani, L., et al. 2006, MNRAS, 371, 821

Mukai, K., Hellier, C., Madejski, G., Patterson, J., \& Skillman, D. R. 2003, ApJ, 597,479

Mulchaey, J. S., Koratkar, A., Ward, M. J., et al. 1994, ApJ, 436, 586

Murakami, H., Baba, H., Barthel, P., et al. 2007, PASJ, 59, 369

Mushotzky, R. F., Winter, L. M., McIntosh, D. H., \& Tueller, J. 2008, ApJL, in press [arXiv: astro-ph/0807.4695]

Nandra, K., \& Iwasawa, K. 2007, MNRAS, 382, L1

Nandra, K., \& Pounds, K. A. 1994, MNRAS, 268, 405

Nenkova, M., Sirocky, M. M., Ivezić, Ž., \& Elitzur, M. 2008a, ApJ, 685, 147

Nenkova, M., Sirocky, M. M., Nikutta, R., Ivezić, Ž., \& Elitzur, M. 2008b, ApJ, 685,160

Norman, C., \& Scoville, N. 1988, ApJ, 332, 124

Onaka, T., Matsuhara, H., Wada, T., et al. 2007, PASJ, 59, 401

Panessa, F., Bassani, L., Cappi, M., et al. 2006, A\&A, 455, 173

Pantin, E., Lagage, O.-P., Claret, A., et al. 2005, The Messenger, 119, 25

Piconcelli, E., Jimenez-Bailón, E., Guainazzi, M., et al. 2005, A\&A, 432, 15

Pier, E. A., \& Krolik, J. H. 1993, ApJ, 418, 673

Poglitsch, A., de Graauw, T., Griffin, M., et al. 2006, in COSPAR, Plenary Meeting, 36th COSPAR Scientific Assembly, 36, 215

Press, W. H., Teukolsky, S. A., Vetterling, W. T., \& Flannery, B. P. 1992, Numerical recipes in FORTRAN. The art of scientific computing (Cambridge: University Press), 2nd edn.

Radomski, J. T., Packham, C., Levenson, N. A., et al. 2008, ApJ, 681, 141

Ramos Almeida, C., Pérez García, A. M., Acosta-Pulido, J. A., \& Rodríguez Espinosa, J. M. 2007, AJ, 134, 2006

Ranalli, P., Comastri, A., \& Setti, G. 2003, A\&A, 399, 39

Reynolds, C. S. 1997, MNRAS, 286, 513

Rieke, G. H., \& Lebofsky, M. J. 1985, ApJ, 288, 618

Risaliti, G. 2002, A\&A, 386, 379

Risaliti, G., Maiolino, R., \& Salvati, M. 1999, ApJ, 522, 157

Risaliti, G., Elvis, M., \& Nicastro, F. 2002, ApJ, 571, 234

Rodriguez Espinosa, J. M., Rudy, R. J., \& Jones, B. 1987, ApJ, 312, 555

Sanders, D. B., Phinney, E. S., Neugebauer, G., Soifer, B. T., \& Matthews, K. 1989, ApJ, 347, 29

Sazonov, S., Revnivtsev, M., Krivonos, R., Churazov, E., \& Sunyaev, R. 2007, A\&A, 462, 57

Schartmann, M., Meisenheimer, K., Camenzind, M., et al. 2008, A\&A, 482, 67

Severgnini, P., Risaliti, G., Marconi, A., Maiolino, R., \& Salvati, M. 2001, A\&A, 368, 44

Shi, Y., Rieke, G. H., Hines, D. C., et al. 2006, ApJ, 653, 127

Siebenmorgen, R., Haas, M., Pantin, E., et al. 2008, A\&A, 488, 83

Soifer, B. T., Bock, J. J., Marsh, K., et al. 2003, AJ, 126, 143

Spinoglio, L., \& Malkan, M. A. 1989, ApJ, 342, 83

Spinoglio, L., Malkan, M. A., Rush, B., Carrasco, L., \& Recillas-Cruz, E. 1995, ApJ, 453, 616

Steffen, A. T., Strateva, I., Brandt, W. N., et al. 2006, AJ, 131, 2826

Suganuma, M., Yoshii, Y., Kobayashi, Y., et al. 2006, ApJ, 639, 46

Tomono, D., Doi, Y., Usuda, T., \& Nishimura, T. 2001, ApJ, 557, 637

Treister, E., Krolik, J. H., \& Dullemond, C. 2008, ApJ, 679, 140

Tueller, J., Mushotzky, R. F., Barthelmy, S., et al. 2008, ApJ, 681, 113

Ueda, Y., Eguchi, S., Terashima, Y., et al. 2007, ApJ, 664, L79

Vasudevan, R. V., \& Fabian, A. C. 2007, MNRAS, 381, 1235

Vasudevan, R. V., \& Fabian, A. C. 2009, MNRAS, 392, 1124

Véron-Cetty, M.-P., \& Véron, P. 2001, A\&A, 374, 92

Véron-Cetty, M.-P., \& Véron, P. 2006, A\&A, 455, 773

Vignali, C., \& Comastri, A. 2002, A\&A, 381, 834

Vignali, C., Brandt, W. N., \& Schneider, D. P. 2003, AJ, 125, 433

Wilkes, B. J., Mathur, S., Fiore, F., Antonelli, A., \& Nicastro, F. 2001, ApJ, 549, 248

Wilman, R. J., \& Fabian, A. C. 1999, MNRAS, 309, 862 